\title{
Formulation and Optimization of Biodegradable Insulin Loaded
} Nanoparticles

\author{
Ahmed Gamal Eid", Nazim Uddin², Samuel Girgis ${ }^{3}$ \\ School of pharmacy and pharmaceutical sciences, University of Sunderland \\ University Department, \\ Sunderland, State SR1 3SD, United Kingdom \\ a.elsafy177@gmail.com, 00447425215850.
}

School of pharmacy and pharmaceutical sciences, University of Sunderland University Department,

Sunderland, State SR1 3SD, United Kingdom

bg65zj@research.sunderland.ac.uk, 00447824809123.
School of pharmacy and pharmaceutical sciences, University of Sunderland
University Department,
Sunderland, State SR1 3SD, United Kingdom
bg96cd@research.sunderland.ac.uk, 00447404780179

\begin{abstract}
This research aims to provide the diabetic patients with more adequate treatment by designing controlled release insulin loaded nanoparticles prepared by biodegradable polymers. Factors to be studied in this research are the effect of the preparation methods using two different preparation techniques microfluidic / salting out technique which is a new and promising technique and a conventional technique which is double emulsion /solvent evaporation technique. The characterization of the nanoparticles was performed in the terms of size, zeta potential, morphological analysis, IR spectrophotometry, thermal analysis, loading and in vitro release of the nanoparticles. All the formulations are showing nanoparticles in the range from 300 to $400 \mathrm{~nm}$ with a negative zeta potential between -10 and $-20 \mathrm{mV}$. Furthermore, all the nanoparticles are showing smooth spherical shape with chemical and thermal compatibility between the ingredients with encapsulation efficiency that ranges from $42 \%$ to $52 \%$ in all formulations.
\end{abstract}

Key-words: microfluidic, salting out, emulsion, solvent evaporation.

\section{*Corresponding author \\ E-Mail: bg96cd@ research.sunderland.ac.uk (Samuel Girgis).}

\section{Introduction:}

Diabetes mellitus is considered one of the most common and serious diseases in the world which affected about $8.5 \%$ of the adult world population in 2014. Most of the diabetic patients depend on multiple daily insulin injections for the treatment which is inconvenient to use for most of them. This research aims to design controlled release insulin loaded nanoparticles prepared by biocompatible and biodegradable polymers in order to provide the diabetic patients with adequate treatment as a substitute for the daily injection of insulin by 
studying different factors in the preparation process of the nanoparticles and how they affect the physicochemical properties of the nanoparticles ${ }^{[1]}$.

Diabetes mellitus is considered one of the most common and serious diseases in the world which affected about $8.5 \%$ of the adult world population in 2014. Most of the diabetic patients depend on multiple daily insulin injections for the treatment which is inconvenient to use for most of them. This research aims to design controlled release insulin loaded nanoparticles prepared by biocompatible and biodegradable polymers in order to provide the diabetic patients with adequate treatment as a substitute for the daily injection of insulin by studying different factors in the preparation process of the nanoparticles and how they affect the physicochemical properties of the nanoparticles ${ }^{[1]}$.

The first factor to be discussed is the preparation method. Two techniques have been used in the manufacturing of the insulin loaded nanoparticles. The first technique is double emulsion/solvent evaporation technique which is a conventional method that is being used in the preparation of micro and nanoparticles for a long time. Also, this technique is being used in some industries on a large scale. A modified type of this technique has been used in this research which consists of two sequential emulsification steps each includes high homogenization force ${ }^{[2]}$. The first emulsification step is to prepare a primary emulsion w/o in which the insulin is solubilized in the aqueous phase and entrapped inside the oily phase which contains the polymers solubilized in dichloromethane. The second emulsification step is to prepare a w/o/w emulsion by homogenizing the primary emulsion in aqueous phase containing poly vinyl alcohol (PVA) as a stabilizer. The organic solvent used in the oily phase dichloromethane (DCM) which is a toxic volatile organic solvent used to solubilize the polymers is then removed by stirring the emulsion for one day allowing the organic solvent to be evaporated ${ }^{[2]}$.

The second technique is microfluidic/salting out which is a new and promising technique has been recently used in the preparation of nanoparticles. Furthermore, microfluidic devices are simple and easy to use with a better control on the size and poly dispersity (PDI) of the nanoparticles produced ${ }^{[3]}$. Microfluidic technique includes only one step for the preparation of the nanoparticles and allows the control on many parameters. The insulin is solubilized in aqueous phase containing PVA as a stabilizer and the polymers solubilized in acetonitrile as an organic phase are introduced to the microfluidic device at controlled flow rate to produce the nanoparticles only in one step using the minimum amount of acetonitrile which is a toxic organic solvent. The organic solvent is then removed by salting out using sodium borate salt ${ }^{[4]}$. The second factor that has been investigated in this research is the type of polymer used in the preparation of the insulin loaded nanoparticles. Three types of polymers have been used for modifying the physiochemical properties of the nanoparticles. The main polymer used is Poly (D, L-lactide-co-glycolide) PLGA Resomer ${ }^{\circledR}$ RG $503 \mathrm{H}$ which is widely used in the preparation of micro and nanoparticles recently. PLGA is a non-toxic, biocompatible and biodegradable polymer synthesized by esterification of lactic acid and glycolic acid. The hydrophilicity of PLGA can be modified by changing the ratio between lactic and glycolic acid but in this research, we are using PLGA with a ratio of 50:50. The second polymer that been used is Poly (DL-lactideco-caprolactone) which is also a non-toxic, biodegradable and biocompatible polyester with a ratio of 85:15 lactide to caprolactone. Poly (DL-lactide-co-caprolactone) opposes hydrophobic properties and the degradation of the polymer is by hydrolytic cleavage into smaller molecules. The third polymer used is poloxamer 188 which is a nonionic surfactant which consists of a hydrophilic part of polyoxyethylene and a hydrophobic part of polyoxypropylene. Poloxamer 188 can act as a co-emulsifier in the preparation process and the hydrophilicity of the polymer may increase the porosity of the nanoparticles and improve the release of insulin ${ }^{[5]}$. 
Six formulations have been prepared in this research, three formulations by each technique and each one was triplicated to provide a significant amount of data to be analyzed. The first type of insulin nanoparticles was prepared by PLGA as the only polymer for the matrix formation. PLGA insulin loaded nanoparticles are for comparing with the other formulations prepared which act as a control for this experiment. The second type of nanoparticles was prepared by physical mixing of PLGA and $5 \%$ of Poly (DL-lactide-co-caprolactone) and the third type of nanoparticles was prepared by the physical mixing of PLGA with $5 \%$ of Poly (DL-lactide-co-caprolactone) and $5 \%$ of poloxamer 188. These nanoparticles are characterized in the terms of of size, zeta potential, morphological analysis, IR spectrophotometry, thermal analysis, loading and in vitro release of the nanoparticles.

\section{Materials and methods}

\subsection{Materials}

Recombinant human insulin was purchased from (Sigma-Aldrich, UK) with a molecular weight of 5800 Da. PLGA Resomer ${ }^{\circledR}$ RG 503 H, Poly(D, L-lactide-co-glycolide) acid terminated with lactide : glycolide ratio 50:50 and molecular weight of 24,000-38,000 Da, Poly (DL-lactide-co-caprolactone) with a ratio of 85:15 lactide: caprolactone DL-lactide 86 $\%$, Poloxamer 188 solution $10 \%$ and Poly(vinyl alcohol) with molecular weight of 13,000 23,000 Da were also purchased from (Sigma-Aldrich, UK). Pierce BCA protein assay kit was purchased from (Thermo Fisher Scientific, UK). Acetonitrile and Dichloromethane were of HPLC grade and the other reagents were analytical grade or higher and table (1) showing the prepared formulations and composition.

Table (1): table showing different types of formulations manufactured by different polymers and techniques

\begin{tabular}{|c|c|c|c|c|c|}
\hline \multirow[b]{2}{*}{$\begin{array}{l}\text { Formulation } \\
\text { ID }\end{array}$} & \multirow[b]{2}{*}{$\begin{array}{c}\text { Technique } \\
\text { used }\end{array}$} & \multicolumn{4}{|c|}{ Composition (mg) } \\
\hline & & $\begin{array}{c}\text { PLGA Resomer } 8 \\
\text { RG 503 H }\end{array}$ & $\begin{array}{l}\text { Poly (D, L-lactide- } \\
\text { co-caprolactone) }\end{array}$ & $\begin{array}{c}\text { poloxamer } \\
188\end{array}$ & Insulin \\
\hline S1 DE & \multirow{9}{*}{$\begin{array}{l}\text { Double } \\
\text { emulsion / } \\
\text { solvent } \\
\text { evaporation }\end{array}$} & 200 & & & 4 \\
\hline $\mathrm{S} 2 \mathrm{DE}$ & & 200 & & & 4 \\
\hline $\mathrm{S} 3 \mathrm{DE}$ & & 200 & & & 4 \\
\hline $\mathrm{S} 4 \mathrm{DE}$ & & 190 & 10 & & 4 \\
\hline S5 DE & & 190 & 10 & & 4 \\
\hline S6 DE & & 190 & 10 & & 4 \\
\hline S7 DE & & 180 & 10 & 10 & 4 \\
\hline S8 DE & & 180 & 10 & 10 & 4 \\
\hline S9 DE & & 180 & 10 & 10 & 4 \\
\hline S1 MF & \multirow{9}{*}{$\begin{array}{l}\text { Microfluidic } \\
\text { /salting out }\end{array}$} & 200 & & & 4 \\
\hline S2 MF & & 200 & & & 4 \\
\hline S3 MF & & 200 & & & 4 \\
\hline S4 MF & & 190 & 10 & & 4 \\
\hline S5 MF & & 190 & 10 & & 4 \\
\hline S6 MF & & 190 & 10 & & 4 \\
\hline S7 MF & & 180 & 10 & 10 & 4 \\
\hline S8 MF & & 180 & 10 & 10 & 4 \\
\hline S9 MF & & 180 & 10 & 10 & 4 \\
\hline
\end{tabular}




\subsection{Methodology}

\subsubsection{Preparation of insulin-loaded nanoparticles using double emulsion / solvent} evaporation technique.

Double emulsion is a well-known technique that is widely used for the preparation of nanoparticles. A modified double emulsion technique has been used in this method which includes preparing a primary dispersion of the drug in organic phase then further emulsification to incorporate the dispersed particles into bigger particles as w/o/w as shown in figure (1).
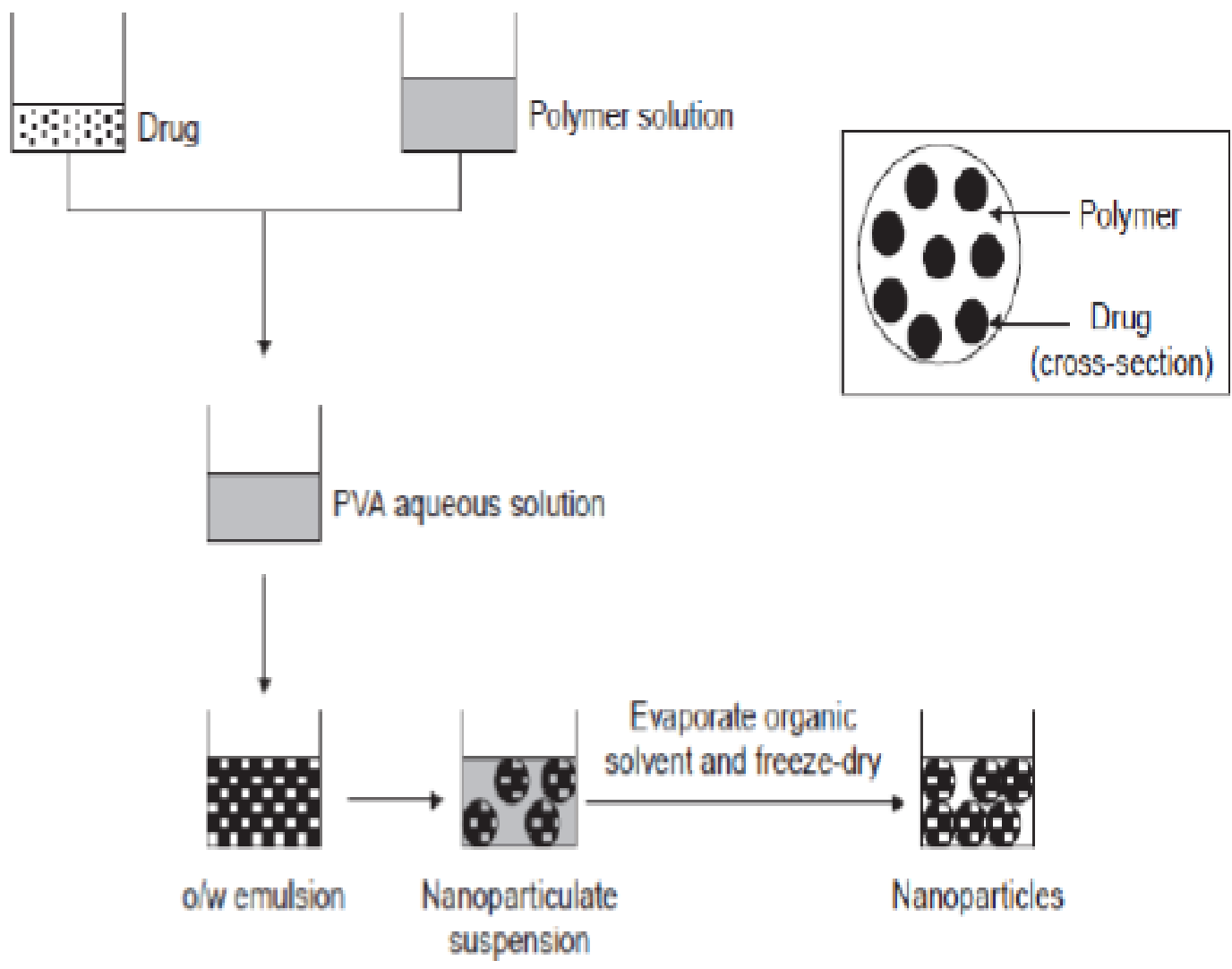

Figure (1): showing a diagram for double emulsion technique ${ }^{[6]}$.

As shown in figure (2) The aqueous phase was prepared by dissolving $4 \mathrm{mg}$ of insulin in 0.5 $\mathrm{ml}$ of $0.1 \mathrm{M} \mathrm{HCl}$ (as insulin has better solubility at lower $\mathrm{pH} 2-2.5 \mathrm{pH}$ ) in Eppendorf tube then $0.5 \mathrm{ml}$ of $2.5 \%$ PVA was added with shaking with vortex (whirlimixer, fisher scientific) till complete dissolving of insulin. For the organic phase $200 \mathrm{mg}$ of polymers as shown in figure (1) were added to $6 \mathrm{ml}$ of dichloromethane with stirring till complete dissolving of the polymers. The primary emulsification was performed by adding the aqueous phase drop wise to the organic phase and homogenization at $6000 \mathrm{rpm}$ for 2 minutes. Then the secondary emulsification was performed adding the primary (W/O) emulsion to $50 \mathrm{ml}$ of $1.25 \%$ PVA as external phase while homogenization at $10000 \mathrm{rpm}$ for 6 minutes using (Silverson homogenizer, $\mathrm{UK})$ to form $(\mathrm{W} / \mathrm{O} / \mathrm{W})$ emulsion. Then evaporation of dichloromethane by stirring the system for $24 \mathrm{hrs}$. After that centrifugation for 15 minutes at $6000 \mathrm{rpm}$ using centrifuge (Mse mistral 1000, UK) and the water was removed then washed for three times to remove the residues of the organic solvent. After that samples were freeze dried using freeze dryer (VirTis BenchTop K series, USA). 


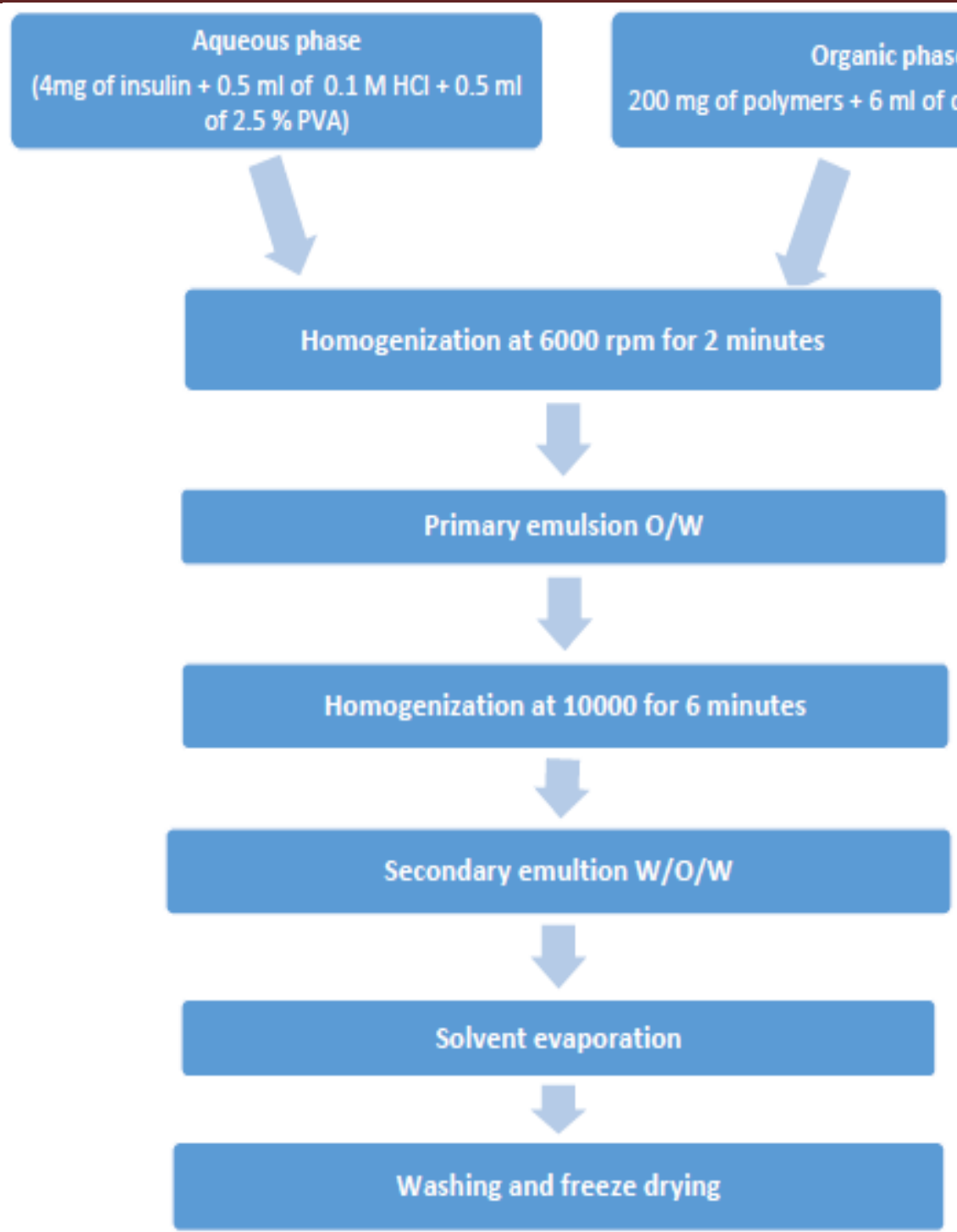

Figure (2): Flow chart showing manufacturing steps of insulin loaded nanoparticles using double emulsion technique.

\subsubsection{Preparation of insulin-loaded nanoparticles using microfluidic technique:}

The aqueous phase was prepared by weighing $4 \mathrm{mg}$ of insulin in Eppendorf tube then $0.5 \mathrm{ml}$ of $0.1 \mathrm{M} \mathrm{HCl}$ was added with shaking using vortex mixer (Whirlimixer, fisher scientific, UK) till complete dissolution of the insulin. Then, $0.5 \mathrm{ml}$ of $2.5 \%$ PVA solution was added and mixed using vortex mixer to make sure insulin is completely dissolved. For the organic phase, $4 \mathrm{ml}$ of acetonitrile were added to $200 \mathrm{mg}$ of the polymers in a beaker while stirring using magnetic stirrer to dissolve the polymers. After that, NanoAssemblr Benchtop instrument (Precision NanoSystems, Inc., Vancouver, BC, Canada) was used to manufacture the nanoparticles with TFR (total flow rate) of $12 \mathrm{ml} / \mathrm{min}$ which controls the mixing speed of the two phases in the microfluidic cartridge, and FRR (flow rate ratio) of 4:1 organic to aqueous phase as shown in figure (3). The resulting nanoparticles were stirred with sodium borate salt to remove acetonitrile. Then our system was centrifuged using centrifuge (Mse mistral 1000, UK) and the water was removed then washed to make sure all residues specially the organic solvent are completely removed. After that the nanoparticles were freeze dried using (VirTis BenchTop K series, USA) ${ }^{[7]}$. 
Polymers solubilized in organic phase
Insulin solubilized in $2.5 \%$ PVA solution

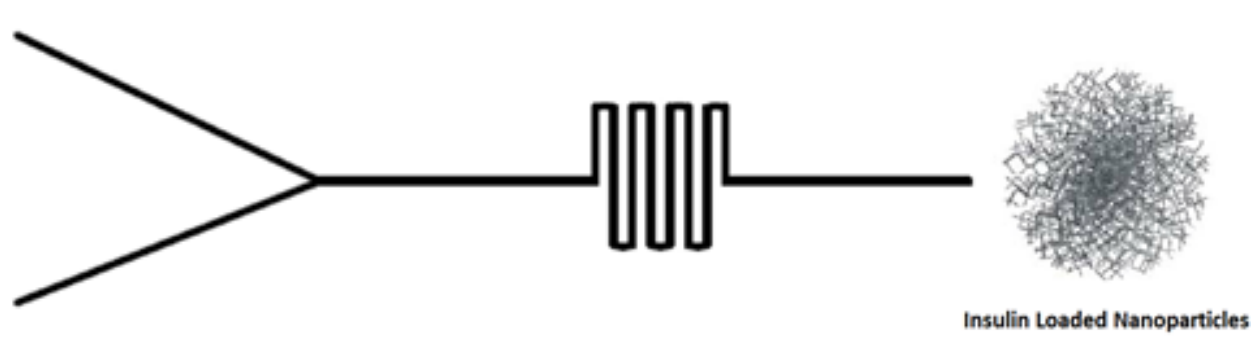

Figure (3): Showing the preparation process of the insulin loaded nanoparticles by microfluidic technique

\subsubsection{Particle size characterization}

The particle size of the nanoparticles is measured using Zetasizer Nano ZS® (Malvern Instruments Limited, UK). Samples were taken from each formula before freeze drying then diluted with purified water to a suitable concentration and mixed using vortex mixer (Whirlimixer, fisher scientific) to suspend the nanoparticles. Then the size is measured using DLS technique (dynamic light scattering). The principle of this technique is fine particles are in constant random Brownian motion, the speed of the particles is related to the particle size at constant temperature. Small particles diffuse faster than large ones. Illuminating the particles produced by the speckle pattern with a laser is observed to measure the speed of the diffusion. By time the intensity of the scattering will fluctuate. Which can be detected by avalanche photodiode detector (APD). The changes in the intensity are then analysed with a digital autocorrelator that produces a correlation function. Then the curve produced can be analysed to give the size and size distribution shown in figure (4). Zetasizer Nano ZS® is easy to use and highly automated with high sensitivity. In addition, samples for size can be measured with little or no dilution ${ }^{[8]}$.

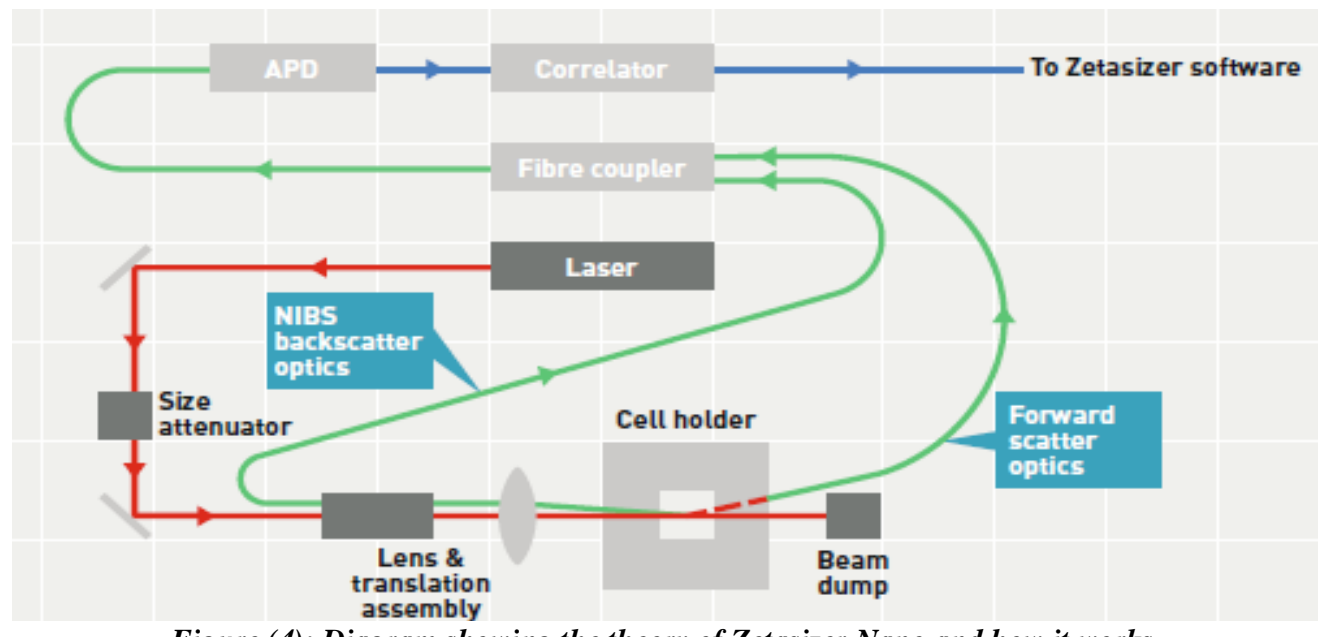

Figure (4): Diagram showing the theory of Zetasizer Nano and how it works.

\subsubsection{Zeta potential.}

Zeta potential is the charge acquired by the particles in specific medium and can also be defined as the electrostatic potential at the electrical double layer that surrounds a nanoparticle in a solution as described in figure (5). Zeta potential was measured using Zetasizer Nano ZS® (Malvern Instruments Limited, UK). Samples were taken from each formula before the freeze drying step then diluted using $0.01 \mathrm{M} \mathrm{KCl}$ solution for better conductivity. Zeta potential is measured by applying an electric field to a particles dispersion or a solution then the velocity of the particles is then measured by applying a patented laser interferometric technique calls M3-PALS (phase analysis light scattering) which enables to calculate the electrophoretic mobility from which zeta potential can be measured ${ }^{[9]}$. 


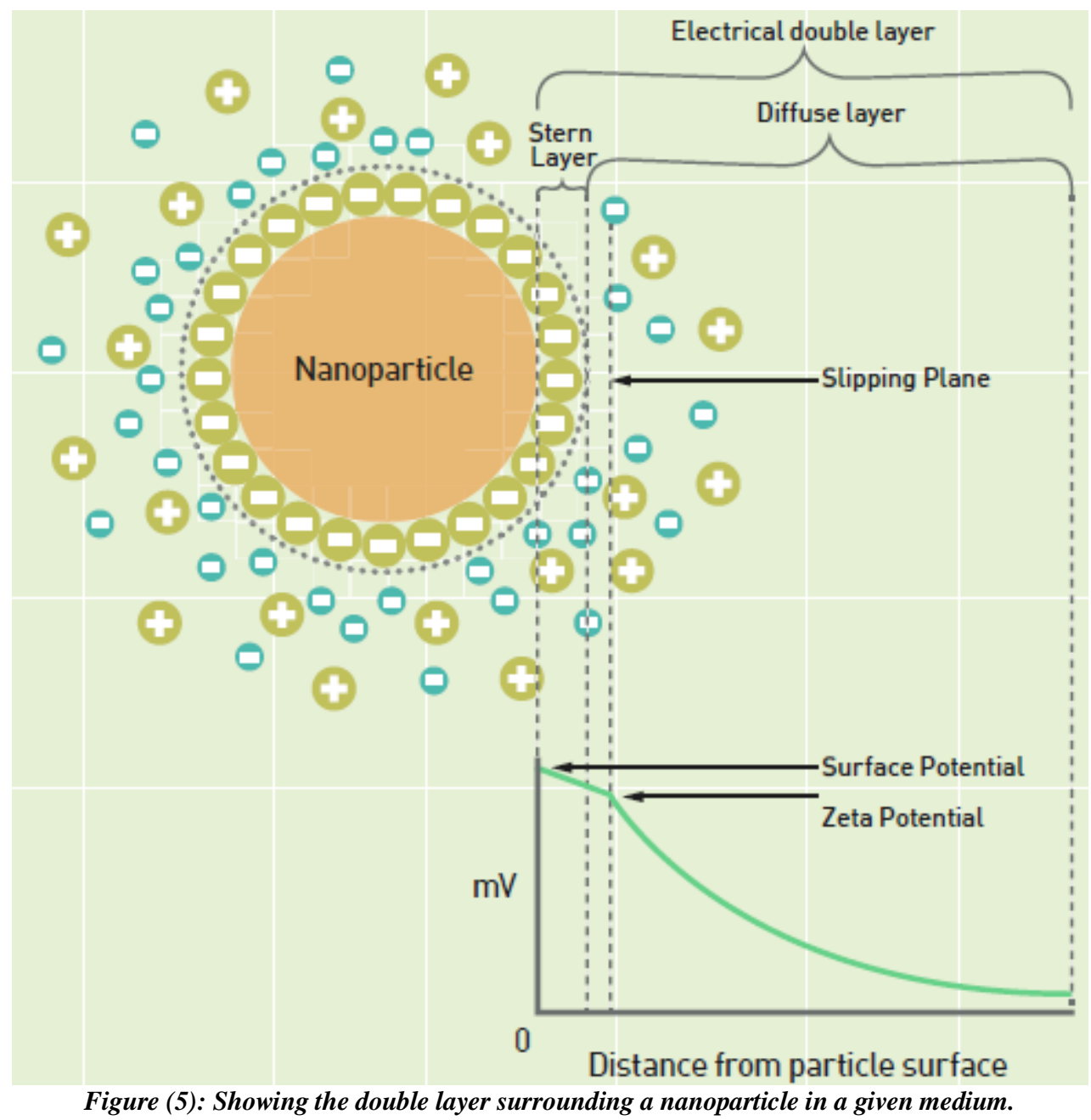

\subsubsection{Morphological analysis and imaging.}

Imaging was performed using scanning electron microscope (SEM). Freeze dried samples were coated with a mixture of Au/Pd using (Quorum SC7620 Sputter Coater) to improve the image quality. Then imaging was performed using scanning electron microscope SEM (S$3000 \mathrm{~N}$, Hitachi, Japan) which can take images for the samples up to 50,000 x. imaging of the samples is very useful to determine the size of the nanoparticles and their distribution.

\subsubsection{Loading of insulin and encapsulation efficiency.}

Insulin loading was performed using direct extraction method of insulin from the nanoparticles. $5 \mathrm{mg}$ of insulin were weighed in Eppendorf tube then $0.5 \mathrm{ml}$ of $1 \mathrm{M} \mathrm{NaOH}$ and incubated at $37^{\circ} \mathrm{C}$ for 24 hours to destroy the nanoparticles and allow the release of the encapsulated insulin. Then, samples were neutralized by $1 \mathrm{M} \mathrm{HCl}$ and centrifuged for 5 minutes at $10000 \mathrm{rpm}$ then supernatant withdrawn for analysis and treated by Pierce ${ }^{\mathrm{TM}}$ BCA Protein Assay Kit which is a detergent-compatible formulation based on bicinchromic acid (BCA) used as a calorimetric method to detect and quantify the total protein. This method is a combination between reduction of $\mathrm{Cu}+2$ to $\mathrm{Cu}+1$ by protein in the alkaline medium with the highly selective and sensitive detection of $\mathrm{Cu}+1$ cation by the use of a reagent that contains bicinchoninic acid. This reaction produces a purple color that results from the chelation of one cuprous ion with two molecules of BCA. The water-soluble complex produced has a high absorbance at $562 \mathrm{~nm}$ and gives almost a linear line by increasing the concentration of the 
protein in the working range from 20 to $2000 \mu \mathrm{g} / \mathrm{mL}$. Development of the color is continuous but it slows down after incubation allowing samples to be assayed at the same time.

Then the absorbance of the samples was measured using xMark $^{\mathrm{TM}}$ Microplate Absorbance Spectrophotometer (Bio-Rad, USA) at $562 \mathrm{~nm}$ then the concentration of the samples was measured by comparing the absorbance of samples with insulin standard calibration curve in the range from 0 to $200 \mu \mathrm{g} / \mathrm{ml}$ which gives us the actual loading of insulin. The encapsulation efficiency is then calculated as the ratio between the actual loading and theoretical loading.

\subsubsection{In vitro release of insulin loaded nanoparticles:}

In vitro release test was performed by adding $5 \mathrm{mg}$ of insulin into $1 \mathrm{ml}$ phosphate buffer (PBS pH 7.4) and incubated at $37{ }^{\circ} \mathrm{C}$. Samples were withdrawn at predetermined time intervals 2 hours, 1 day, 2 days, 3 days, 4 days and 7 days. Three samples were withdrawn from each formula and replaced with fresh phosphate buffer. Samples withdrawn were analyzed by Pierce ${ }^{\mathrm{TM}}$ BCA Protein Assay Kit then the absorbance was measures by XMark $^{\mathrm{TM}}$ Microplate Absorbance Spectrophotometer (Bio-Rad, USA) at $562 \mathrm{~nm}$ then compared with insulin standard calibration curve in the range from 0 to $200 \mu \mathrm{g} / \mathrm{ml}$.

\subsubsection{Compatibility and stability of insulin loaded nanoparticles:}

Stability and compatibility were assessed by two different methods. The first method is FTIR (Fourier transform infrared spectrophotometry) to assess the chemical stability and the second method is DSC (Differential scanning calorimeter) to assess the thermal behaviour and stability of the nanoparticles which will be discussed as follow.

\subsubsection{FTIR (Fourier transform infrared spectrophotometry).}

Chemical stability and compatibility of freeze dried samples of insulin loaded nanoparticles were measured using Fourier transform infrared spectrophotometer (IRAffinity1S, Shimadzu, Japan). FTIR can detect the functional groups in the chemical substances qualitatively and quantitively. Furthermore, by comparing the spectrum of the samples with their raw materials we can detect any chemical reactions that might happens inside the nanoparticles and assess the compatibility of them.

The insulin loaded nanoparticles were introduced to the FTIR then the resulted spectrums were compared with raw materials spectrums and analyzed using Labsolutions IR software.

\subsubsection{DSC (Differential scanning calorimeter).}

Stability and compatibility were also performed using differential scanning calorimeter (DSC q1000, TA instruments, UK). DSC was used to determine the transition temperature for each formula and its raw materials and compared together. Any change in the transition temperature of the samples over the raw materials indicates incompatibility or instability of the nanoparticles. 3 to 5 grams of samples were loaded to aluminum pans at scanning temperature between 40 to $300{ }^{\circ} \mathrm{C}$. Samples were purged with dry nitrogen and the temperature was raised by $10{ }^{\circ} \mathrm{C} / \mathrm{min}$ with pure indium as a standard.

\section{Results.}

The aim of research was to investigate the effect of manufacturing technique and polymer type on physiochemical properties of insulin loaded nanoparticles e.g. size, zeta potential, shape, stability, encapsulation efficiency and release of insulin.

\subsection{Size:}

Size of the nanoparticles has a great effect on stability, targeting and absorption of the nanoparticles, so it is very important to control and monitor the particles' size. In this section 
size and particles distribution results will be discussed among the different formulations that were made with different types of polymers and techniques. At first, insulin loaded nanoparticles were formulated by double emulsion / solvent evaporation technique as shown in table (2). Nanoparticles manufactured by PLGA Resomer® RG $503 \mathrm{H}$ showed the biggest particle size amongst the other formulations with an average of $343 \mathrm{~nm}$. However, the nanoparticles manufactured by PLGA Resomer® RG $503 \mathrm{H}$, caprolactone copolymer and poloxamer 188 showed the smallest particle size with average of 328 n.m., while the nanoparticles manufactured with PLGA Resomer® RG $503 \mathrm{H}$ and caprolactone copolymer have average of $338 \mathrm{n} . \mathrm{m}$. On the other hand, for nanoparticles manufactured by microfluidic technique as shown in table (3) also exhibited the same pattern as the double emulsion. As for the PLGA nanoparticles they have the biggest particle size with average of $328 \mathrm{n} . \mathrm{m}$. then comes the PLGA and caprolactone copolymer nanoparticles with an average of 321 n.m, finally comes the PLGA, caprolactone copolymer and poloxamer nanoparticles with the smallest size of 317 n.m.

For the comparison between different techniques, nanoparticles prepared by the microfluidic technique exhibited smaller size than nanoparticles prepared by double emulsion technique for each formulation. Furthermore, all nanoparticles are showing small PDI (polydispersity index) that ranges from 0.15 to 0.35 which indicates low variation in the particle size and homogenous distribution.

Table (2): Particle size and PDI of insulin loaded nanoparticles performed by double emulsion / solvent evaporation technique

\begin{tabular}{|c|c|c|c|c|c|}
\hline \multicolumn{6}{|c|}{ Double emulsion / solvent evaporation Technique } \\
\hline Formulation ID & Size $n . m$ & PDI & Polymer type & $\begin{array}{l}\text { size } \mathrm{nm} \\
\text { (mean) }\end{array}$ & $\begin{array}{l}\text { PDI } \\
\text { avg }\end{array}$ \\
\hline S1 DE & 330 & 0.14 & \multirow{3}{*}{ PLGA NPS } & \multirow{3}{*}{$343 \pm 33$} & \multirow{3}{*}{0.22} \\
\hline S2 DE & 357 & 0.29 & & & \\
\hline S3 DE & 340 & 0.23 & & & \\
\hline S4 DE & 310 & 0.12 & \multirow{3}{*}{$\begin{array}{c}\text { PLGA NPs }+5 \% \text { caprolactone } \\
\text { copolymer }\end{array}$} & \multirow{3}{*}{$339 \pm 28$} & \multirow{3}{*}{0.15} \\
\hline S5 DE & 365 & 0.2 & & & \\
\hline S6 DE & 317 & 0.12 & & & \\
\hline S7 DE & 320 & 0.22 & \multirow{3}{*}{$\begin{array}{c}\text { PLGA NPs }+5 \% \text { caprolactone } \\
\text { copolymer }+5 \% \text { poloxamer } 188\end{array}$} & \multirow{3}{*}{$328 \pm 38$} & \multirow{3}{*}{0.19} \\
\hline S8 DE & 320 & 0.19 & & & \\
\hline S9 DE & 353 & 0.16 & & & \\
\hline
\end{tabular}

Table (3): Particle size and PDI of insulin loaded nanoparticles performed by microfluidic / salting out technique

\begin{tabular}{|c|c|c|c|c|c|}
\hline \multicolumn{6}{|c|}{ Microfluidic / salting out technique } \\
\hline Formulation ID & Size $n . m$ & PDI & Polymer type & $\begin{array}{l}\text { Size } \mathrm{nm} \\
\text { (mean) }\end{array}$ & $\begin{array}{l}\text { PDI } \\
\text { avg }\end{array}$ \\
\hline S1 MF & 281 & 0.20 & \multirow{3}{*}{ PLGA NPS } & \multirow{3}{*}{$328 \pm 39$} & \multirow{3}{*}{0.26} \\
\hline $\mathrm{S} 2 \mathrm{MF}$ & 366 & 0.27 & & & \\
\hline S3 MF & 337 & 0.31 & & & \\
\hline S4 MF & 307 & 0.24 & \multirow{3}{*}{$\begin{array}{c}\text { PLGA NPs }+5 \% \text { caprolactone } \\
\text { copolymer }\end{array}$} & \multirow{3}{*}{$321 \pm 27$} & \multirow{3}{*}{0.26} \\
\hline S5 MF & 336 & 0.27 & & & \\
\hline S6 MF & 351 & 0.29 & & & \\
\hline S7 MF & 268 & 0.14 & \multirow{3}{*}{$\begin{array}{l}\text { PLGA NPs }+5 \% \text { caprolactone } \\
\text { copolymer+ } 5 \% \text { poloxamer } 188\end{array}$} & \multirow{3}{*}{$317 \pm 59$} & \multirow{3}{*}{0.31} \\
\hline S8 MF & 332 & 0.44 & & & \\
\hline S9 MF & 379 & 0.35 & & & \\
\hline
\end{tabular}




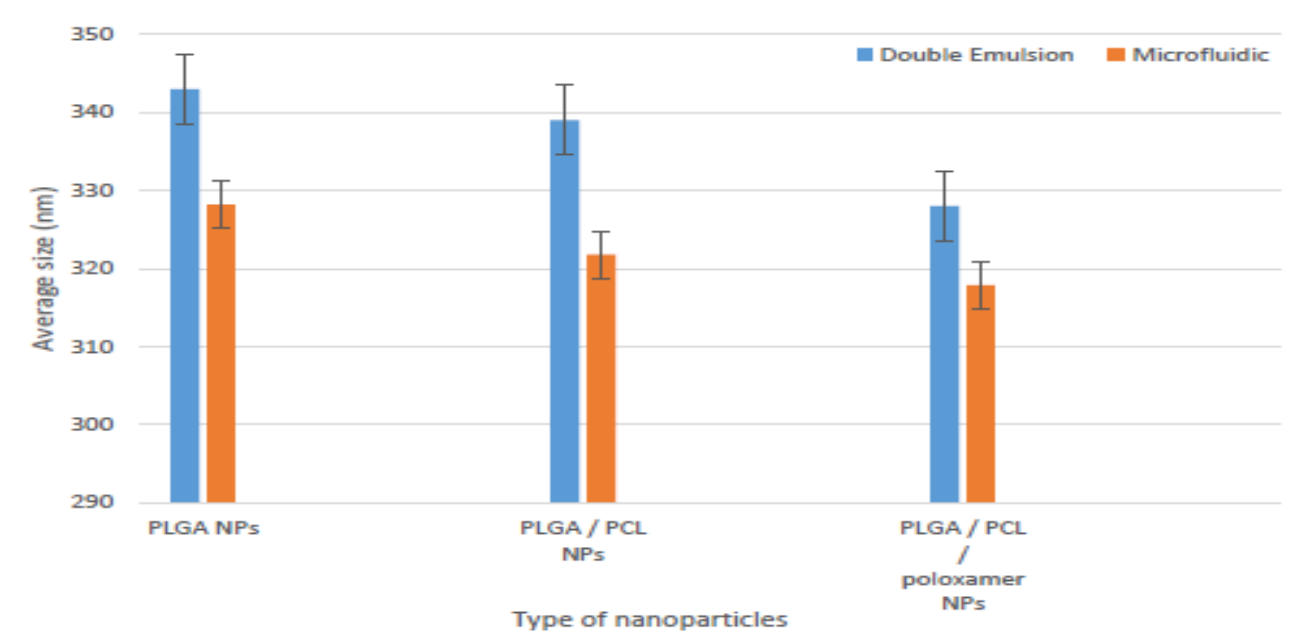

Figure(6): Graph showing results for average size of insulin loaded nanoparticles performed by both double emulsion/solvent evaporation and microfluidic/salting out technique

Table (4): Table showing results for zeta potential of insulin loaded nanoparticles performed by double emulsion / solvent evaporation technique

\begin{tabular}{|c|c|c|c|c|}
\hline \multicolumn{5}{|c|}{ Double emulsion / solvent evaporation technique } \\
\hline Formulation ID & Z.P. $\mathrm{mV}$ & Polymer type & $\begin{array}{l}\text { Z.P. avg } \\
\mathrm{mV}\end{array}$ & $\begin{array}{l}\text { Standard } \\
\text { Deviation }\end{array}$ \\
\hline S1 DE & -11.6 & \multirow{3}{*}{ PLGA NPS } & \multirow{3}{*}{-10.6} & \multirow{3}{*}{1.3} \\
\hline S2 DE & -9.0 & & & \\
\hline S3 DE & -11.3 & & & \\
\hline S4 DE & -10.2 & \multirow{3}{*}{ PLGA NPs + $5 \%$ caprolactone copolymer } & \multirow{3}{*}{-11.7} & \multirow{3}{*}{1.8} \\
\hline S5 DE & -13.1 & & & \\
\hline S6 DE & -11.9 & & & \\
\hline S7 DE & -11.3 & \multirow{3}{*}{$\begin{array}{c}\text { PLGA NPs }+5 \% \text { caprolactone copolymer } \\
+5 \% \text { poloxamer } 188\end{array}$} & \multirow{3}{*}{-8.1} & \multirow{3}{*}{2.4} \\
\hline S8 DE & -6.0 & & & \\
\hline S9 DE & -6.9 & & & \\
\hline
\end{tabular}

Table (5): Table showing results for zeta potential of insulin loaded nanoparticles performed by microfluidic / salting out technique

\begin{tabular}{|c|c|c|c|c|}
\hline \multicolumn{5}{|c|}{ Microfluidic/salting out technique } \\
\hline Sample Name & Z.P. $\mathrm{mV}$ & Polymer type & $\begin{array}{l}\text { Z.P. avg } \\
\mathrm{mV}\end{array}$ & $\begin{array}{l}\text { Standard } \\
\text { Deviation }\end{array}$ \\
\hline S1 MF & -15.8 & \multirow{3}{*}{ PLGA NPS } & \multirow{3}{*}{-13.2} & \multirow{3}{*}{3.2} \\
\hline $\mathrm{S} 2 \mathrm{MF}$ & -9.2 & & & \\
\hline S3 MF & -14.6 & & & \\
\hline S4 MF & -12.1 & \multirow{3}{*}{ PLGA NPs + $5 \%$ caprolactone copolymer } & \multirow{3}{*}{-12.1} & \multirow{3}{*}{1.3} \\
\hline S5 MF & -11.9 & & & \\
\hline $\mathrm{S} 6 \mathrm{MF}$ & -12.5 & & & \\
\hline S7 MF & -16.6 & \multirow{3}{*}{$\begin{array}{c}\text { PLGA NPs }+5 \% \text { caprolactone copolymer } \\
+5 \% \text { poloxamer } 188\end{array}$} & \multirow{3}{*}{-14.8} & \multirow{3}{*}{4.4} \\
\hline $58 \mathrm{MF}$ & -9.2 & & & \\
\hline S9 MF & -18.7 & & & \\
\hline
\end{tabular}




\subsection{Zeta potential}

Zeta potential is a very important parameter for the nanoparticles which plays important role in stability and targeting of the nanoparticles. Each sample has been measured six times and average was taken for each. However, As shown in table (4) and (5) all the nanoparticles are showing a negative charge because of PLGA polymer in the range from -6 to $-19 \mathrm{~m} . \mathrm{v}$. Starting with the particles manufactured by double emulsion / solvent evaporation technique the particles prepared from PLGA polymer only have zeta potential average of $-10.6 \mathrm{~m} . \mathrm{v}$ while PLGA with caprolactone copolymer nanoparticles and PLGA with caprolactone copolymer and poloxamer 188 have average zeta potential of $-11.7 \mathrm{~m} . \mathrm{v}$ and $-10.8 \mathrm{~m} . \mathrm{v}$ respectively. These nanoparticles manufactured by double emulsion technique are showing almost the same value of zeta potential. For the nanoparticles prepared by microfluidic technique the nanoparticles prepared by PLGA with caprolactone copolymer and poloxamer 188 show the highest value of zeta potential with an average of $-18.1 \mathrm{mV}$ followed by PLGA nanoparticles and PLGA with caprolactone nanoparticles with average values of $-13.2 \mathrm{mv}$ and $12.13 \mathrm{mV}$ respectively.

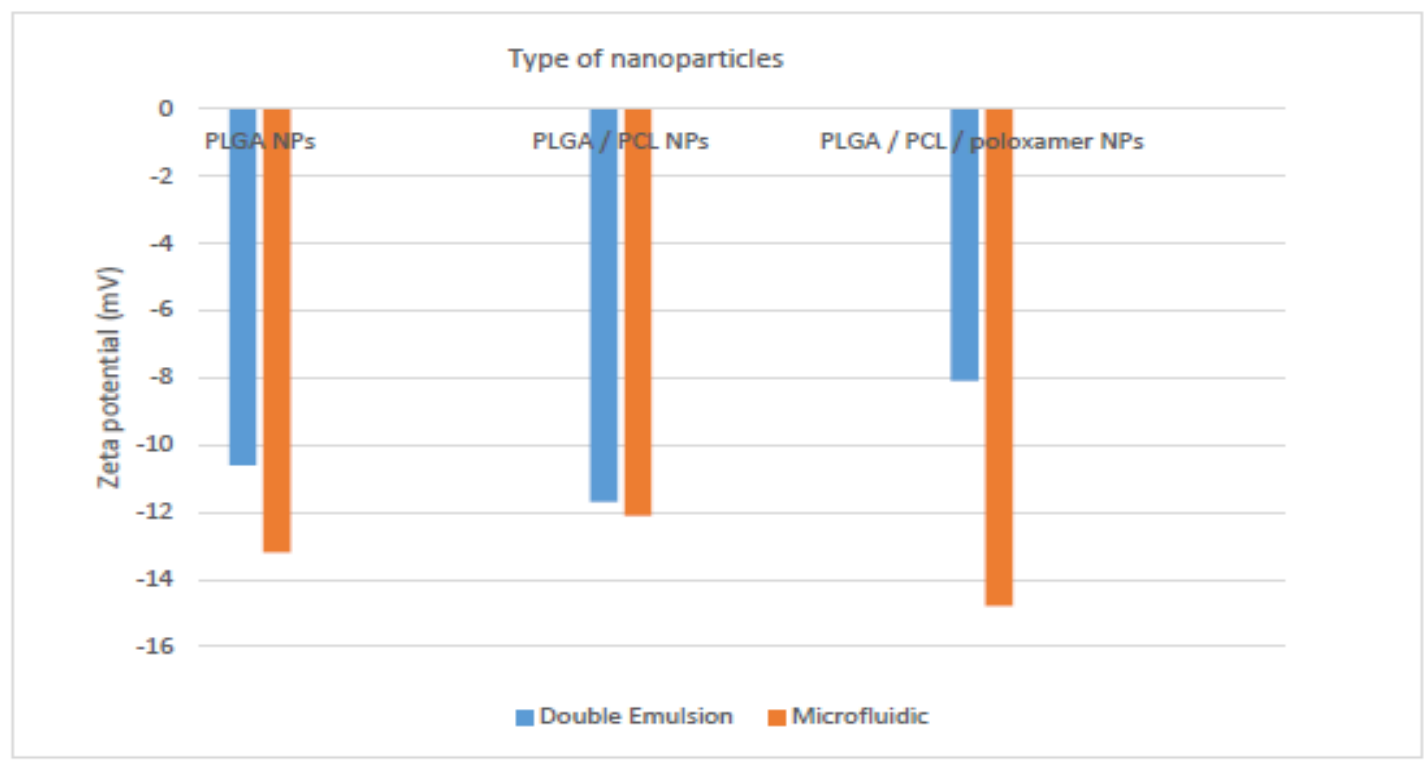

Figure(7): Graph showing results for average zeta potential of insulin loaded nanoparticles performed by both double emulsion / solvent evaporation and microfluidic / salting out technique

On the other hand, nanoparticles prepared by microfluidic technique are showing higher values of zeta potential than those prepared by double emulsion technique for each formulation.

\subsection{Morphological analysis and imaging.}

Morphological analysis for the nanoparticles is important for assessing the quality of the nanoparticles according to the shape, size and surface. All the nanoparticles are showing smooth spherical shape with average size between 300 and $400 \mathrm{~nm}$. By comparing the different types of nanoparticles, nanoparticles prepared by microfluidic technique are showing smaller size and more homogenous distribution and low variation in size as shown in figure $(9,11,13)$. On the other hand, nanoparticles prepared by double emulsion technique are showing slightly bigger particles and more variation in size as shown in figure $(8,10,12)$ comparing to those prepared by microfluidic technique. 


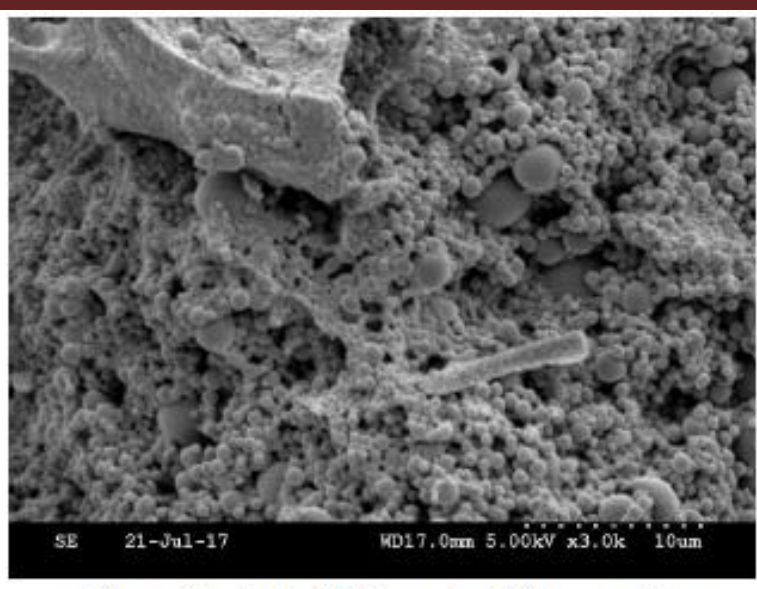

Figure (8): showing SEM image for PLGA nanoparticles prepared by double emulsion technique

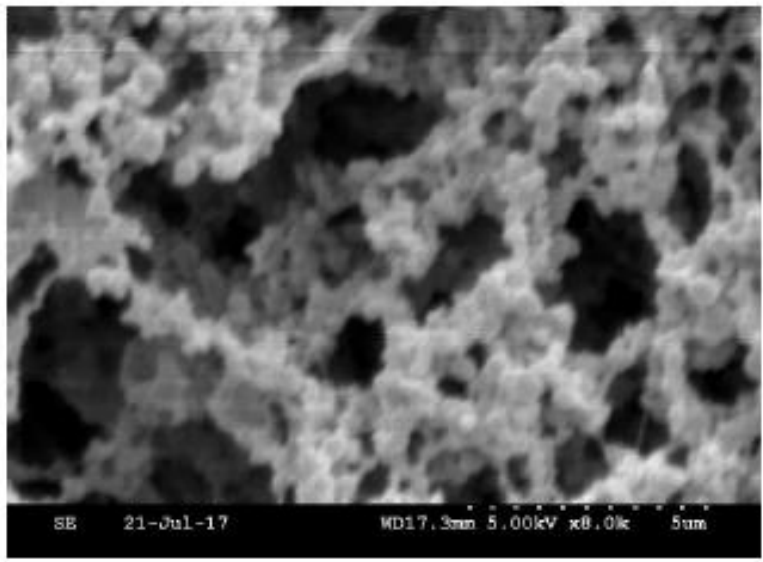

Figure (10): showing SEM image for PLGA / caprolactone nanoparticles prepared by double emulsion technique

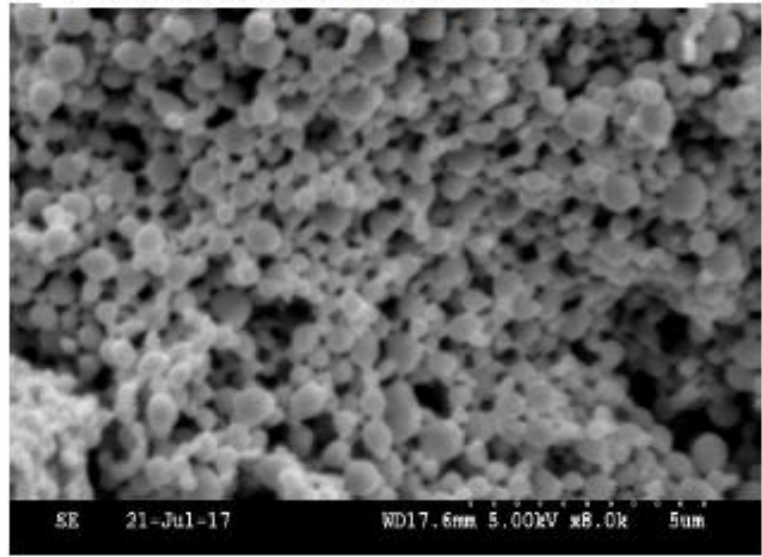

Figure (12): Showing SEM image for PLGA / caprolactone / poloxamer 188 nanoparticles prepared by double emulsion

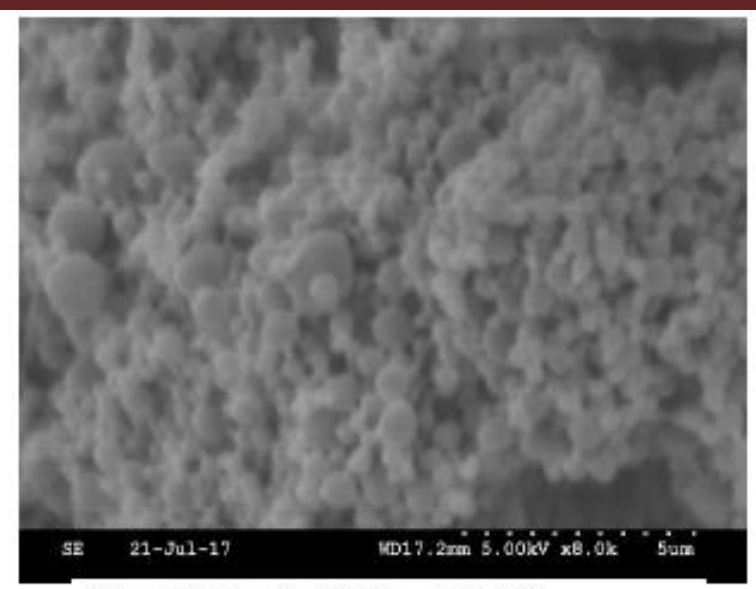

Figure (9): showing SEM image for PLGA nanoparticles prepared by microfluidic technique

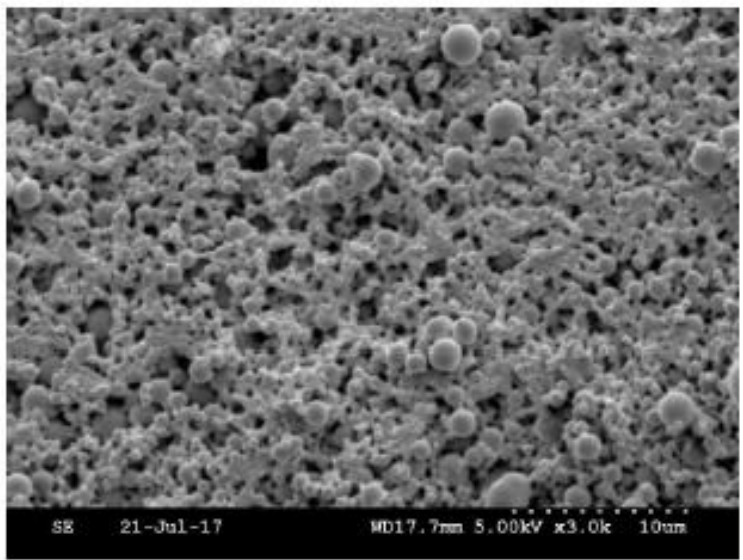

Figure (11): showing SEM image for PLGA/

caprolactone nanoparticles prepared by microfluidic

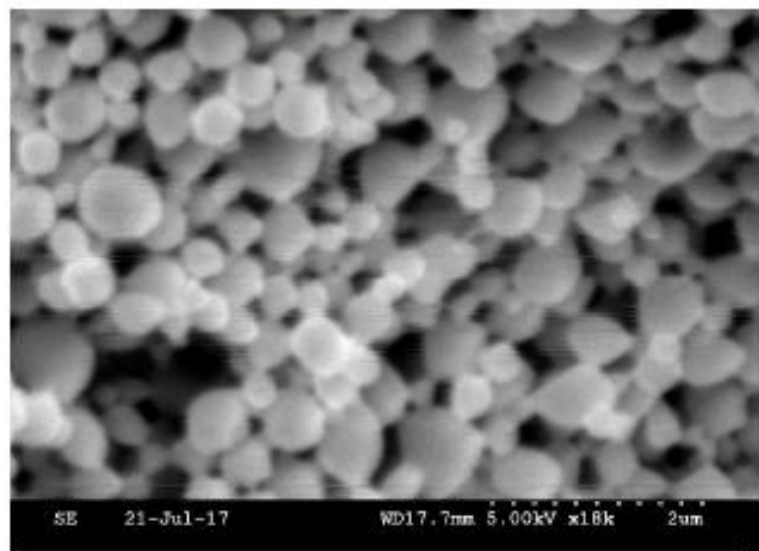

Figure (13): Showing SEM image for PLGA / caprolactone / poloxamer 188 nanoparticles prepared by microfluidic technique

\subsection{Loading of insulin and encapsulation efficiency}

Loading of nanoparticles and encapsulation efficiency give indication if the preparation method is efficient or not. Each sample was measured three times and the average was calculated. However, as shown in table (6) and (7) the encapsulation efficiency of the nanoparticles manufactured by double emulsion technique are showing slightly higher values 
for the encapsulation efficiency more than the microfluidic technique. For the nanoparticles prepared by double emulsion technique, PLGA nanoparticles have the higher encapsulation value with an average of $52.74 \%$, then come PLGA with caprolactone copolymer nanoparticles and poloxamer 188 nanoparticles and PLGA with caprolactone copolymer with average encapsulation of $45.11 \%$ and $42.38 \%$ respectively. For the nanoparticles prepared by microfluidic technique, PLGA with caprolactone copolymer nanoparticles and poloxamer 188 nanoparticles have the higher encapsulation value then PLGA and caprolactone copolymer nanoparticles and PLGA nanoparticles with the values $44.15 \%, 43.14 \%$ and $40.52 \%$.

Table (6): Showing results for release and encapsulation efficiency of insulin loaded nanoparticles

\begin{tabular}{|c|c|c|c|c|c|c|c|c|}
\hline $\begin{array}{l}\text { Formulation } \\
\text { ID }\end{array}$ & $\begin{array}{c}\text { Abs } \\
1\end{array}$ & $\begin{array}{c}\text { Abs } \\
2\end{array}$ & $\begin{array}{c}\text { Abs } \\
3\end{array}$ & $\begin{array}{l}\text { Abs } \\
\text { avg. }\end{array}$ & $\begin{array}{l}\text { conc. } \\
\mu / \mathrm{mg}\end{array}$ & $\begin{array}{c}\text { Encapsulatio } \\
\text { n } \%\end{array}$ & Polymer type & $\begin{array}{l}\text { Encapsulation } \\
\text { efficiency \% }\end{array}$ \\
\hline S1 DE & 0.11 & 0.11 & 0.09 & 0.10 & 9.1 & 45.4 & \multirow{3}{*}{ PLGA NPs } & \multirow{3}{*}{$52.7=15.8$} \\
\hline $\mathrm{S} 2 \mathrm{DE}$ & 0.15 & 0.16 & 0.15 & 0.15 & 13.7 & 68.5 & & \\
\hline $\mathrm{S} 3 \mathrm{DE}$ & 0.10 & 0.10 & 0.10 & 0.10 & 8.9 & 44.3 & & \\
\hline $\mathrm{S} 4 \mathrm{DE}$ & 0.09 & 0.10 & 0.10 & 0.10 & 8.5 & 42.6 & \multirow{3}{*}{$\begin{array}{l}\text { PLGA / } \\
\text { caprolactone } \\
\text { copolymer } \\
\text { NPs }\end{array}$} & \multirow{3}{*}{$42.4 \pm 1.6$} \\
\hline $\mathrm{S} 5 \mathrm{DE}$ & 0.11 & 0.10 & 0.10 & 0.10 & 8.8 & 44.0 & & \\
\hline S6 DE & 0.08 & 0.10 & 0.09 & 0.09 & 8.1 & 40.5 & & \\
\hline S7 DE & 0.11 & 0.11 & 0.10 & 0.11 & 9.3 & 46.6 & \multirow{3}{*}{$\begin{array}{c}\text { PLGA / } \\
\text { capprolactone } \\
\text { copolymer / } \\
\text { poloxamer 188 } \\
\text { NPs }\end{array}$} & \multirow{3}{*}{$45.1 \pm 1.5$} \\
\hline S\& DE & 0.10 & 0.11 & 0.10 & 0.10 & 9.2 & 46.1 & & \\
\hline $\mathrm{S} 9 \mathrm{DE}$ & 0.10 & 0.09 & 0.09 & 0.10 & 8.5 & 42.6 & & \\
\hline
\end{tabular}

Table (7): Showing results for release and encapsulation efficiency of insulin loaded nanoparticles manufactured by microfluidic/salting out technique

\begin{tabular}{|c|c|c|c|c|c|c|c|c|}
\hline $\begin{array}{c}\text { Formulation } \\
\text { ID }\end{array}$ & $\begin{array}{c}\text { Abs } \\
1\end{array}$ & $\begin{array}{c}\text { Abs } \\
2\end{array}$ & $\begin{array}{c}\text { Abs } \\
3\end{array}$ & $\begin{array}{l}\text { Abs } \\
\text { avg. }\end{array}$ & $\begin{array}{l}\text { conc. } \\
\mu / m g\end{array}$ & $\begin{array}{c}\text { Encapsulation } \\
\%\end{array}$ & Polymer type & $\begin{array}{c}\text { Encapsulation } \\
\text { efficiency \% }\end{array}$ \\
\hline S1 MF & 0.09 & 0.09 & 0.09 & 0.09 & 8.0 & 40.1 & \multirow{3}{*}{ PLGA NPs } & \multirow{3}{*}{$40.5 \pm 0.8$} \\
\hline S2 MF & 0.09 & 0.09 & 0.10 & 0.09 & 8.3 & 41.3 & & \\
\hline S3 MF & 0.09 & 0.08 & 0.10 & 0.09 & 8.0 & 40.2 & & \\
\hline S4 MF & 0.10 & 0.10 & 0.10 & 0.10 & 8.7 & 43.5 & \multirow{3}{*}{$\begin{array}{c}\text { PLGA/ } \\
\text { caprolactone } \\
\text { copolymer NPs }\end{array}$} & \multirow{3}{*}{$43.1 \pm 1.7$} \\
\hline S5 MF & 0.09 & 0.10 & 0.09 & 0.09 & 8.2 & 41.1 & & \\
\hline S6 MF & 0.10 & 0.10 & 0.10 & 0.10 & 9.0 & 44.8 & & \\
\hline S7 MF & 0.09 & 0.11 & 0.11 & 0.10 & 9.2 & 45.8 & \multirow{3}{*}{$\begin{array}{c}\text { PLGA / } \\
\text { caprolactone } \\
\text { copolymer / } \\
\text { poloxamer 188 } \\
\text { NPs }\end{array}$} & \multirow{3}{*}{$44.2 \pm 1.6$} \\
\hline SS MF & 0.10 & 0.10 & 0.10 & 0.10 & 8.9 & 44.5 & & \\
\hline S9 MF & 0.09 & 0.10 & 0.09 & 0.10 & 8.4 & 42.2 & & \\
\hline
\end{tabular}

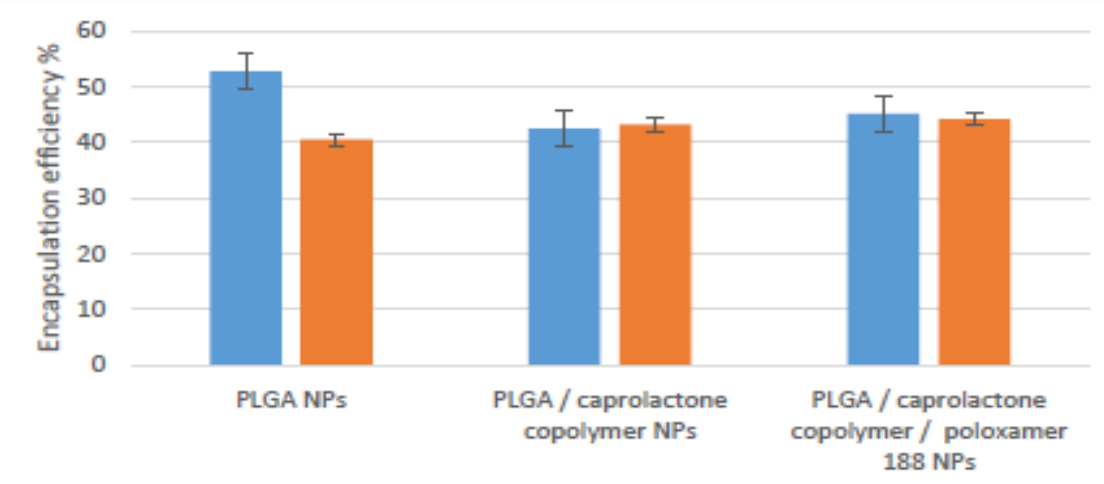

Type of nanoparticles

$\square \mathrm{DE}$ MF

Figure(14): Graph showing the encapsulation efficiency of insulin loaded nanoparticles performed by both double emulsion / solvent evaporation and microfluidic / salting out techniques 


\subsection{In vitro release of insulin loaded nanoparticles.}

In vitro release of nanoparticles was performed by incubation the samples for 7 days at $37{ }^{\circ} \mathrm{C}$ then analyzed using Pierce ${ }^{\mathrm{TM}}$ BCA Protein Assay Kit and measured with microplate reader at $562 \mathrm{~nm}$ at time intervals 2 hours, 1 day, 2 days, 3days, 4days and 7 days. However, for the double emulsion technique as shown in figure (15) PLGA nanoparticles are showing the highest exhibits the highest burst release with $70.32 \%$ after 2 hours and a full release of $93.25 \%$ after 7 days. On the other hand, nanoparticles incorporated with caprolactone are showing much lower burst with $37.89 \%$ after 2 hours and full release with $48.48 \%$ after 7 days which is almost similar to the PLGA nanoparticles with caprolactone copolymer and poloxamer 188 with initial burst release of $36.26 \%$ and full release of $47.07 \%$ after 7 days. For the nanoparticles prepared by microfluidic technique as shown if figures (16), they are showing much lower initial burst release than nanoparticles prepared by double emulsion technique. However, PLGA nanoparticles are showing initial burst release with $12.29 \%$ after 2 hours and full release of $57.64 \%$ after seven days. furthermore, PLGA nanoparticles incorporated with caprolactone copolymer and PLGA nanoparticles with caprolactone copolymer and poloxamer 188 exhibit initial release of $10.12 \%$ and $29.04 \%$ after 2 hours and a full release of $41.04 \%$ and $43.45 \%$ after seven days respectively.

Table (8): Showing in vitro cumulative release of insulin nanoparticles over seven days prepared by double emulsion / solvent evaporation technique

\begin{tabular}{|l|c|c|c|c|c|c|}
\hline \multicolumn{7}{|c|}{ Double emulsion / solvent evaporation technique } \\
\hline & $\mathbf{2}$ hrs & 1 day & 2 days & 3 days & 4 days & 7 days \\
\hline PLGA NPs & $70.3 \%$ & $80.8 \%$ & $81.8 \%$ & $80.8 \%$ & $84.6 \%$ & $93.3 \%$ \\
\hline $\begin{array}{l}\text { PLGA / caprolactone copolymer } \\
\text { NPs }\end{array}$ & $37.8 \%$ & $47.2 \%$ & $47.2 \%$ & $49.3 \%$ & $47.7 \%$ & $48.5 \%$ \\
\hline $\begin{array}{l}\text { PLGA / caprolactone copolymer / } \\
\text { poloxamer 188 NPS }\end{array}$ & $36.2 \%$ & $44.4 \%$ & $44.4 \%$ & $44.2 \%$ & $42.0 \%$ & $47.1 \%$ \\
\hline
\end{tabular}

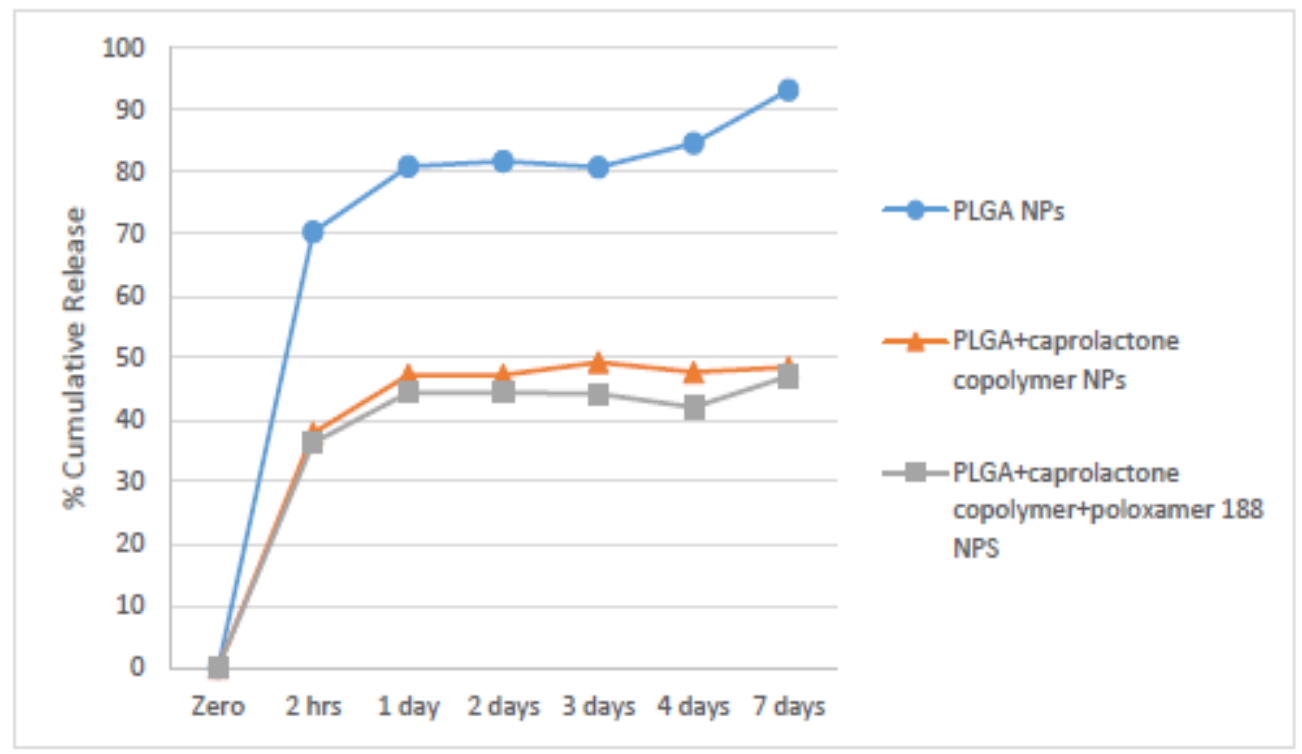

Figure (15): a graph showing in vitro cumulative release of insulin nanoparticles over seven days prepared by double emulsion / solvent evaporation technique 
Table (9): Showing in vitro cumulative release of insulin nanoparticles over seven days prepared by microfluidic / salting out technique

\begin{tabular}{|l|c|c|c|c|c|c|}
\hline \multicolumn{7}{|c|}{ Micro fluidic / salting out technique } \\
\hline & $\mathbf{2}$ hrs & 1 day & 2 days & 3 days & 4 days & 7 days \\
\hline PLGA NPs & $12.2 \%$ & $37.5 \%$ & $36.5 \%$ & $38.4 \%$ & $42.4 \%$ & $57.6 \%$ \\
\hline $\begin{array}{l}\text { PLGA / caprolactone } \\
\text { copolymer NPs }\end{array}$ & $10.1 \%$ & $30.9 \%$ & $29.1 \%$ & $30.9 \%$ & $43.7 \%$ & $41.0 \%$ \\
\hline $\begin{array}{l}\text { PLGA caprolactone } \\
\text { copolymer / poloxamer 188 } \\
\text { NPS }\end{array}$ & $29.0 \%$ & $35.9 \%$ & $32.9 \%$ & $35.9 \%$ & $49.9 \%$ & $43.5 \%$ \\
\hline
\end{tabular}

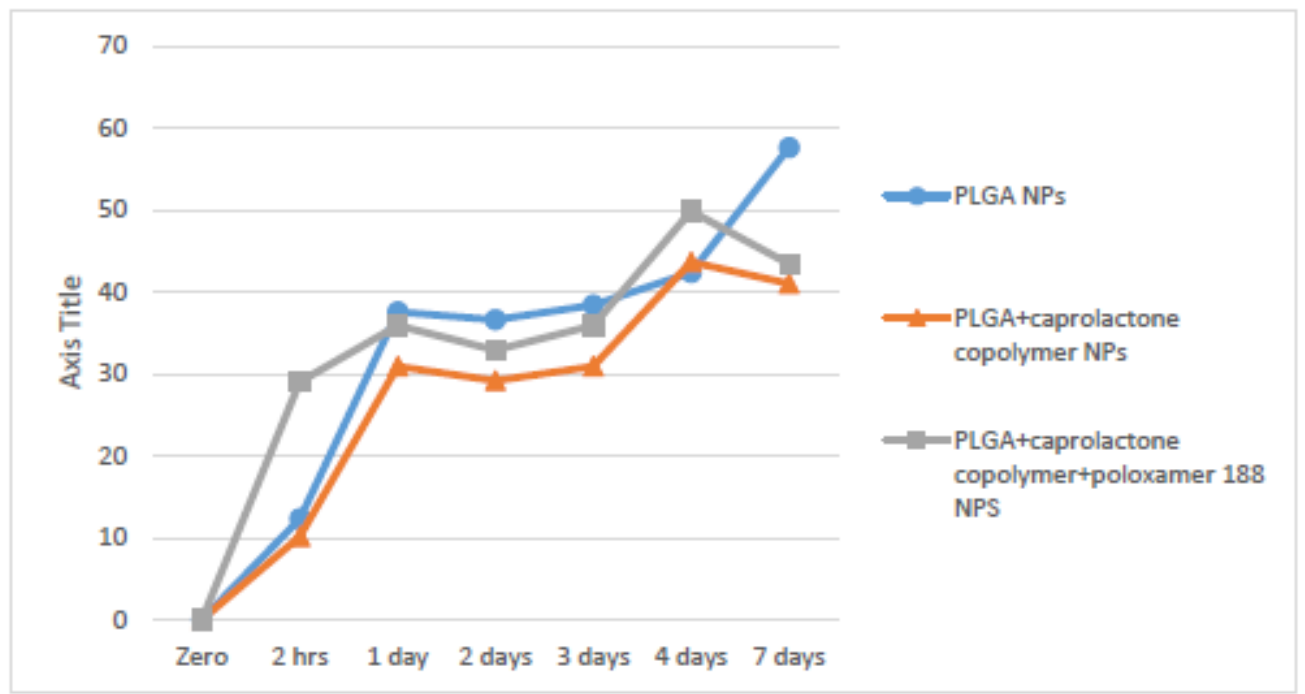

Figure (16): A graph showing in vitro cumulative release of insulin nanoparticles over seven days prepared by microfluidic / salting out technique

\subsection{Stability and compatibility of insulin loaded nanoparticles.}

\subsubsection{FTIR (Fourier transform infrared spectrophotometry):}

Chemical stability was performed by identifying the functional groups for the freezedried nanoparticles formulations and their raw materials using FTIR. However, PLGA is the main polymer in these formulations which shows a stretching peak of (C-O-C) group at 1088 $\mathrm{cm}-1$, and a methyl stretching $(\mathrm{C}-\mathrm{H})$ between 1400 and $1500 \mathrm{~cm}-1$, main peak of stretching carbonyl group (C-O-O) between 1700 and $1800 \mathrm{~cm}-1$ and stretching $(\mathrm{CH}, \mathrm{CH} 2, \mathrm{CH} 3)$ groups between 2800 and $3000 \mathrm{~cm}-1$. For the nanoparticles prepared by PLGA for both double emulsion and microfluidic techniques as shown in figure $(17,18)$, the IR chart for the nanoparticles was plotted against insulin, PVA, PLGA and the chart showing matching between the nanoparticles and PLGA charts and disappearing of other peaks for insulin and PVA which means all the materials are encapsulated inside the PLGA nanoparticles.

Also for the nanoparticles prepared by PLGA with carpolactone copolymer for both double emulsion and microfluidic as shown in figure $(19,20)$ the nanoparticles chart was plotted against insulin, PLGA, PVA and carpolactone copolymer are showing matching between nanoparticles and PLGA and for the nanoparticles prepared by PLGA with carpolactone copolymer and poloxamer 188 for both double emulsions and microfluidic as shown in figure $(21,22)$, the nanoparticles have the same peaks as PLGA polymer. 
All the nanoparticles charts are matching with PLGA chart and other peaks disappeared and this indicates full encapsulation all the maerials inside the nanoparticles.

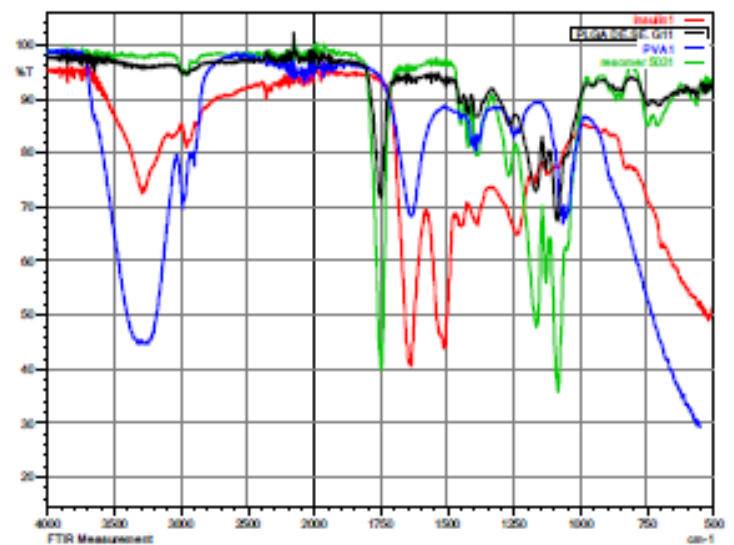

Figure (17): Showing FTIR chart for PLG.4 NPs and raw materials used in the proparation by double emulsion tochnique

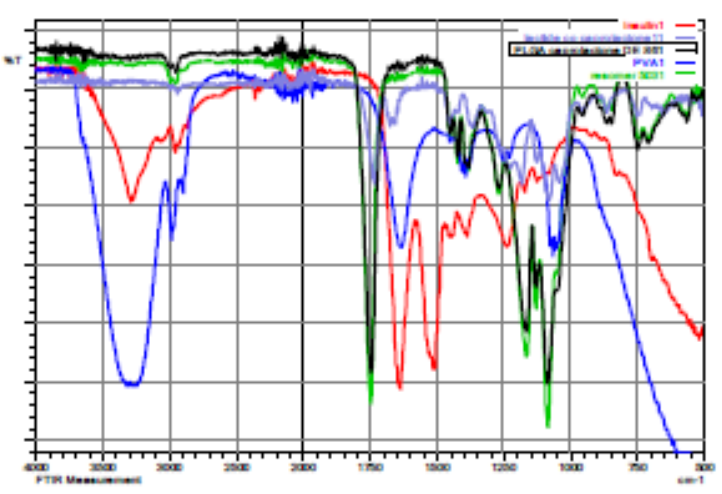

Figure (19): Showing FIIR chant for PLGA / caprolactone NPs and rew materials used in the preparation by double cmulsion

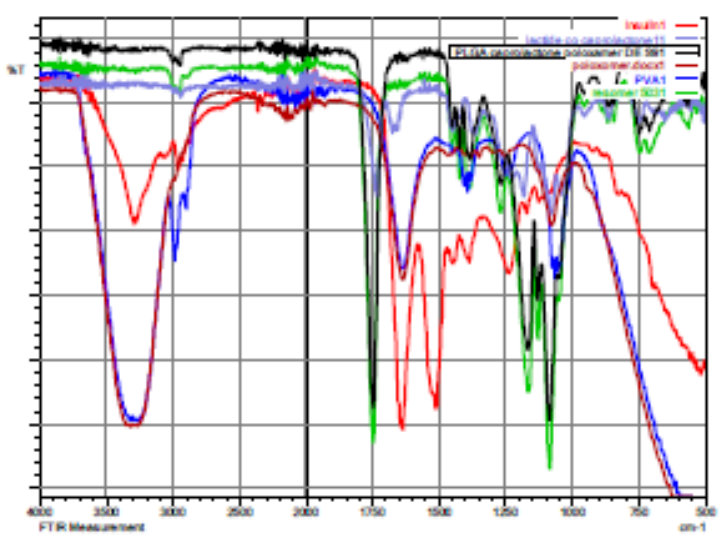

Figure (21): Showing FIIR chart for PLGA / caprolactone / poloxaner $18 S$ NPs and naw materials used in the proparation by double emilsion tochnique

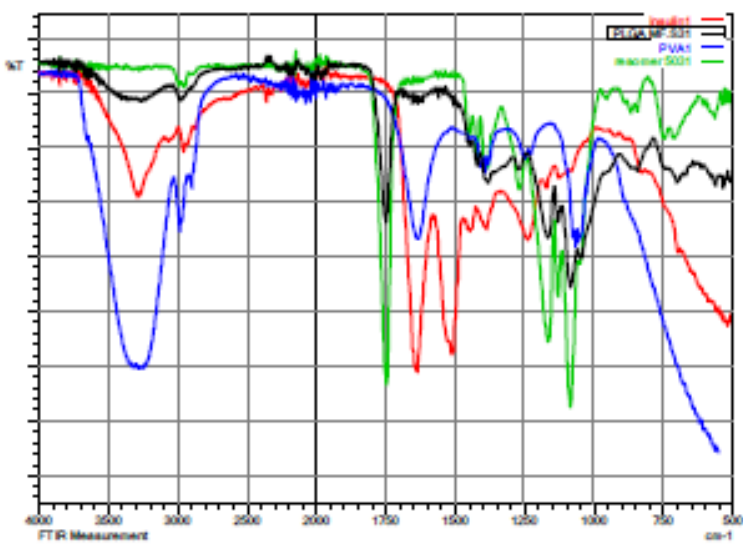

Figure (18): Showing FIIR chart for PLGA NPs and naw materials used in the preparation by microfluidic technique

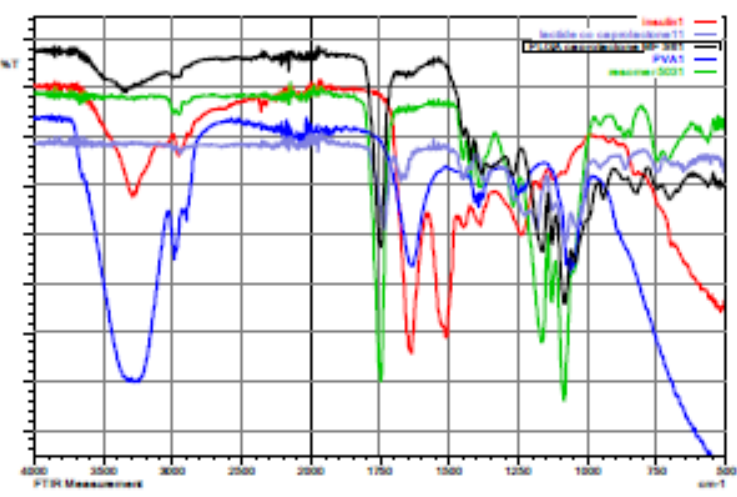

Figure (20): Showing FTIR chant for PLGA / caprolactone NPS and raw materials used in the proparation by microfuidic

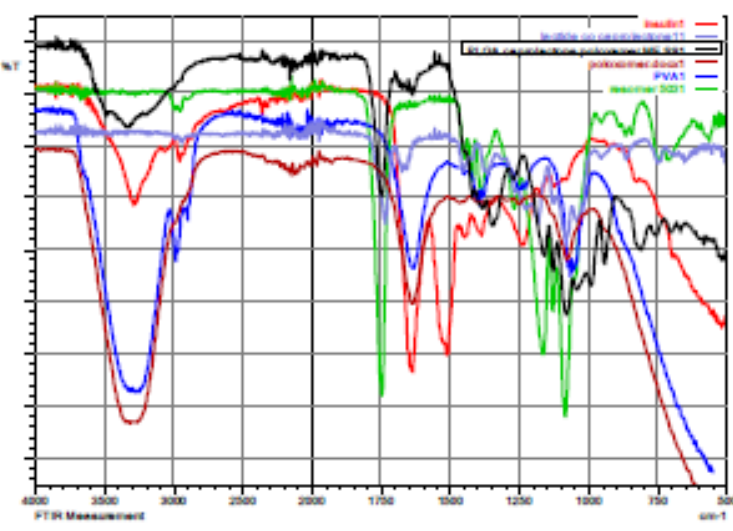

Figure (22): Showing FIIR chan for PLGA / caprolactone / poloxamer $1 S S$ NPs and raw materials used in the preparation by microfluidic tochnique

\subsubsection{DSC (Differential scanning calorimeter).}

Differential scanning calorimeter (DSC) was used to study the thermal properties and thermal stability of the nanoparticles. The nanoparticles thermogram was plotted against their raw materials to compare between them. However, PLGA nanoparticles prepared by double emulsion and microfluidic techniques as shown in figure (23 and 24) are showing a transition temperature $(\mathrm{Tg})$ at about $49{ }^{\circ} \mathrm{C}$ the same as PLGA polymer. Incorporation of carpolactone 
copolymer to the nanoparticles as shown in figure (25 and 26) didn't affect the transition temperature $(\mathrm{Tg})$ which is about $49{ }^{\circ} \mathrm{C}$ the same as PLGA. Also incorporation of poloxamer 188 has no effect on the transition temperature for the nanoparticles as shown in figure (27 and 28) which is $49{ }^{\circ} \mathrm{C}$.

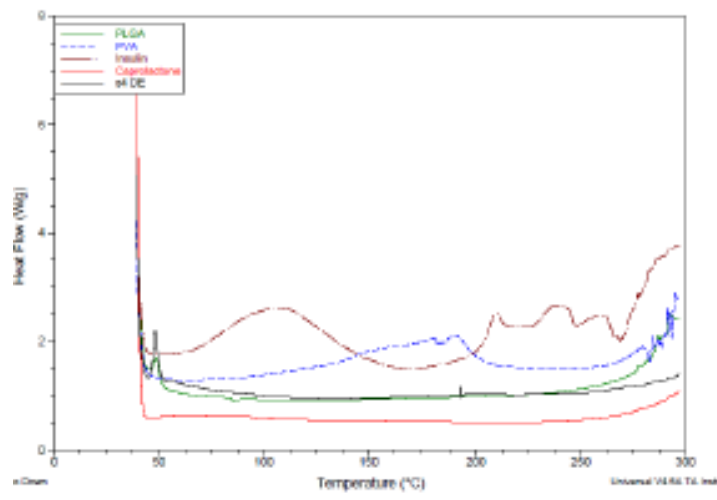

Figure (23): Showing DSC chart for PLGA NPs and now materials used in the preparation by double cmulsion tochique

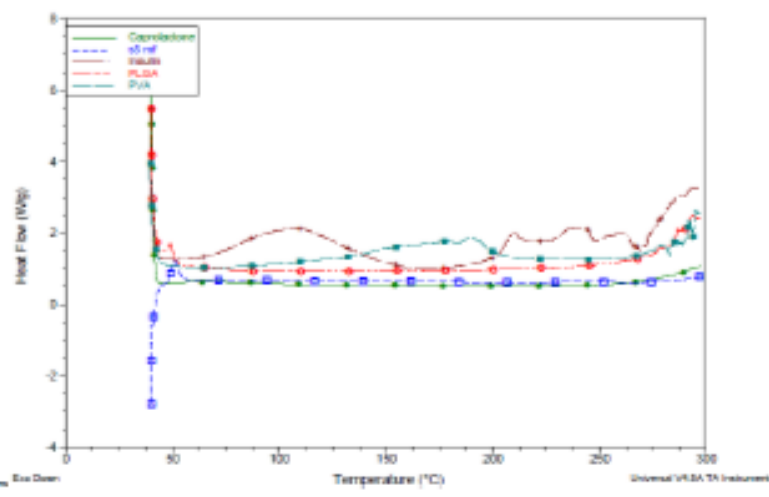

Figure (24): Showing DSC chart for PLG.A NPs and rav materials used in the proparation by microfuidic tochnique

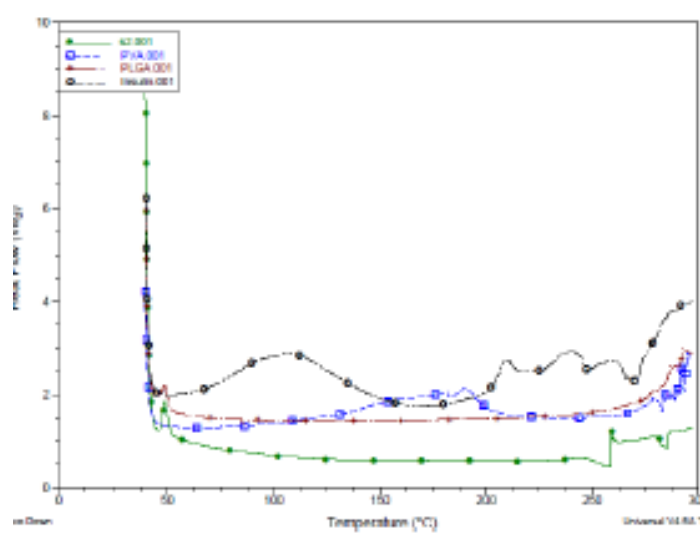

Figure (25): Showing DSC chart for PLGA / caprolactone NPs and raw materials used in the preparation by double emulsion technique

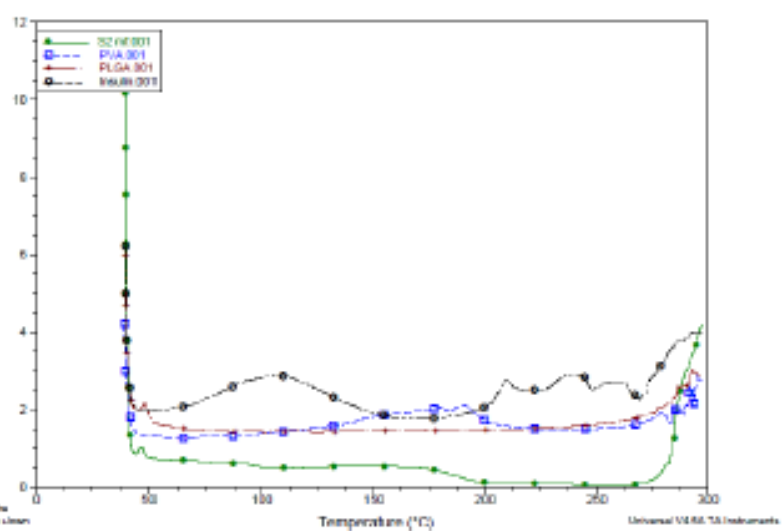

Figure (26): Showing DSC chart for PLGA / caprolactone NPs and naw materials used in the proparation by miarofuidic technique

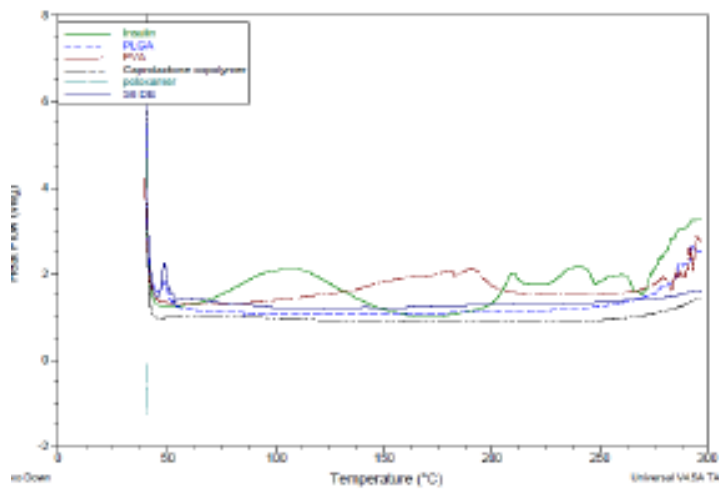

Figure (27): Showing DSC chart for PLG.A / caprolactone / poloxaner 183 NPs and naw materials used in the proparation by double emilsion tochnique

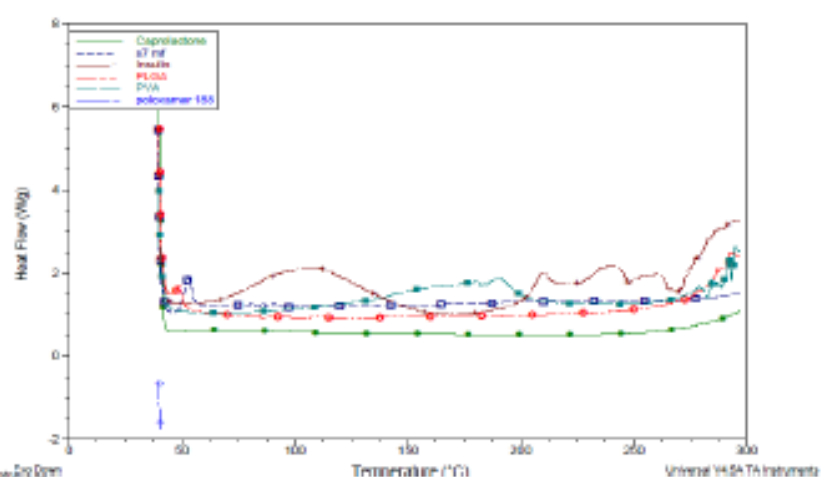

Figure (28): Showing DSC chart for PLGA / caprolactone / poloxamer $18 S$ NPs and row materials used in the proparation by microfluidic technigue 


\section{Discussion.}

In this part, we go to discuss the previous results in details and the effects of different polymers and preparation techniques on the physiochemical properties of the nanoparticles.

\subsection{Method of preparation}

Two different techniques have been used in the preparation of the nanoparticles. The first method is double emulsion / solvent evaporation technique. Emulsions are widely used in the preparation of micro and nanoparticles at large scale in industry. There are many types of emulsions e.g. simple emulsions which are a two-phase dispersion and multiple emulsions which are complex multi-phase dispersions commonly used as intermediate process in the preparation of micro and nanoparticles. Double emulsion is a type of multiple emulsions techniques which has been modified for the preparation of insulin nanoparticles in this research. It consists of two sequential emulsification steps each of them includes a high forced homogenization. Insulin which was solubilized in $2.5 \%$ PVA solution as the aqueous phase and the polymers which were solubilized in acetonitrile were homogenized first to prepare the primary emulsion w/o then further homogenization for the primary emulsion in $1.25 \%$ PVA solution was performed to prepare the secondary emulsion w/o/w. The homogenization steps are associated with random shear distribution which leads to high variation in the droplets size in each step of the preparation. Also, the high homogenization force might lead to the escape of inner droplets from the outer droplets that might leads to low encapsulation efficiency. Also,

The double emulsion consists of many steps in the preparation which consumes a lot of time. Solvent evaporation was applied next to remove the acetonitrile which is a toxic volatile organic solvent by stirring the emulsion for 24 hours at atmospheric temperature or lower which is also a time-consuming method ${ }^{[10]}$.

The second technique used is microfluidic/salting out technique. Recently, microfluidic technique is being used a lot in many researches for the preparation of micro and nanoparticles which works by exploiting controlled mixing of streams in micro-sized channels ${ }^{[11]}$. The formulations were prepared by solubilizing the insulin in $2.5 \%$ PVA solution which is the aqueous phase and the polymers were solubilized in acetonitrile which is the orgonic phase. After that, both phases were introduced to the microfluidic device at a flow rate of $12 \mathrm{ml} / \mathrm{min}$ and flow rate ratio of 1:4 respectively. Microfluidic fluidic technique has a superior control over the size and dispersity of the particles over the double emulsion technique. Furthermore, has the advantage of being easy to use and the parameters like flow rate and flow rate ratio can be easily adjusted using its software. Also, it doesn't consume time as the preparation of each sample took only a few minutes. For the removal of acetonitrile which is a toxic volatile organic solved, salting out method was used by sodium borate which consumes much less time than the solvent evaporation ${ }^{[7]}$.

\subsection{Polymers used in the preparations.}

In this research, the insulin loaded nanoparticles are prepared from a biodegradable polymer PLGA Resomer® RG $503 \mathrm{H}$ which is the main polymer that forms not less than 90 $\%$ of the weight of the prepared particles. In addition, characterization properties were optimized by incorporation of two polymers Poly (DL-lactide-co-caprolactone) and poloxamer 188 in a minor ratio to improve the properties of the nanoparticles.

PLGA Resomer ${ }^{\circledR}$ RG $503 \mathrm{H}$ is a type of Poly (DL-lactide-co-caprolactone) polymers which considered one of the most attractive polymers used now. It is a non-toxic, biocompatible and biodegradable polymer. It is a poly ester consists of poly lactic and poly glycolic acid. Lactic acid is less hydrophilic than glycolide so the ratio between them controls 
the hydrophilicity of the polymer. Also, the molecular weight can be controlled by changing the length of the polymer chain. PLGA degradation is performed by the hydrolysis of the ester bond between lactic and glycolide acid and it exhibits bulk degradation more than surface degradation ${ }^{[6]}$. PLGA is compatible with most of the drugs and considered has a perfect host especially for hydrophilic drugs. Resomer® RG $503 \mathrm{H}$ has a ratio 50:50 between lactic and glycolic acid with moderate hydrophobicity ${ }^{[12]}$.

Poly (DL-lactide-co-caprolactone) shares a lot of properties with PLGA as it is a hydrophilic, biocompatible and biodegradable polymer. It is prepared by the esterification of lactide and caprolactone the ratio between them controls the hydrophilicity of the drug. The type used in this research has a ratio of 85:15 between lactide and caprolactone with high molecular weight. The degradation occurs by hydrolysis of the ester bond to smaller molecules and bulk erosion is more susceptible than surface erosion ${ }^{[13]}$.

Poloxamer 188 is a nonionic surfactant which has a hydrophobic part of polyoxypropylene and a hydrophilic part of polyoxyethylene . poloxamer is a surface active agent so by incorporation poloxamer in the preparation of the nanoparticles it improves the dispersion of the particles by acting as a co-emulsifier and gives a smaller size of nanoparticles. also, the hydrophilicity of the polymer improves the porosity which improve the release behaviour of the nanoparticles.

\subsection{Size.}

Size is a very critical parameter in the nanoparticles formulation. Nanoparticles should be small enough to circulate freely in the circulation system as the smallest capillaries in the human body is around $4 \mu \mathrm{m}$ and to allow the uptake of the nanoparticles to the cells. Furthermore, the small size of the nanoparticles plays important role in avoiding phagocytosis. In addition, small particle size reduces the irritation resulting from the injection and reduces potential irritation reactions. Also, size is very critical for the loading of the drug as by decreasing the size the loading capacity is increased and it is very critical for the release patterns as well. The size of the particles determines the release of the drug as by decreasing the size

We increase the surface area of the nanoparticle and increase the degradation rate. For PLGA and caprolactone polymers bulk erosion is faster than the surface erosion which is depending on the surface area of the particles.

Two different techniques have been used in the preparation double emulsion / solvent evaporation and microfluidic / salting out which have a big influence on the size of the nanoparticles. However, nanoparticles prepared by double emulsion technique have bigger size than nanoparticles prepared by microfluidic technique with a larger distribution and the reason for that multi emulsions techniques like the double emulsion includes multi sequential emulsification steps which are associated with high random shear distribution which leads to broad size distribution of the nanoparticles in each emulsification step. On the other hand, microfluidic is a promising new technique providing the capability of nanoparticles preparation just in one step. Furthermore, particle size and its distribution can be controlled efficiently providing smaller nanoparticles with low PDI.

The polymers used have a non-significant influence on the size of the nanoparticles. Three different polymers have been used in the formulations PLGA Resomer® RG 503 H, Poly (D, L-lactide-co-caprolactone) and Poloxamer 188. The addition of these polymers has been performed by physical mixing. In addition, all these polymers are hydrophobic and have a close molecular weight so the size of the nanoparticles is slightly affected. However, PLGA nanoparticles have the biggest size for both double emulsion and microfluidic. Incorporation of $5 \%$ of caprolactone copolymer by physical mixing to the nanoparticles slightly decreased 
the particle size average for the double emulsion technique around $5 \mathrm{~nm}$ and around $7 \mathrm{~nm}$ for nanoparticles prepared by microfluidic. Addition of $5 \%$ poloxamer 188 also slightly decreased the average size of the nanoparticles by $10 \mathrm{~nm}$ for the double emulsion and $4 \mathrm{~nm}$ for the microfluidic.

\subsection{Zeta potential.}

Zeta potential is the charge acquired by particles in a given medium which represents the surface charge of the particles and it depends on the concentration and the types of ions. Zeta potential is important for obtaining stability in the solution as the particles with similar charges repel and by increasing the charge increase the resistance of the particles for agglomeration. Also, zeta potential is important for the absorption and targeting of the particles as the cell membrane is composed of phospholipids which have a negative charge on the surface. So, by decreasing the negative charge the easier the uptake of the particles. So, zeta potential is very important and critical as it regulates the stability of the solution and the targeting of the particles.

Results of Zeta potential for the insulin nanoparticles are showing that the preparation technique has the biggest influence on the zeta potential as the nanoparticles prepared by microfluidic technique have more negative charge than those prepared by double emulsion technique which is mostly related to the difference in the organic solvent used in each technique as acetonitrile was used as organic solvent for microfluidic technique and dichloromethane was used as an organic solvent for the double emulsion technique.

The different types of polymers used don't have a big influence on zeta potential. For the double emulsion technique comes PLGA with caprolactone nanoparticles with the highest average of zeta potential then PLGA with caprolactone and poloxamer 188 nanoparticles and PLGA nanoparticles respectively. For the microfluidic technique comes the PLGA with caprolactone copolymer and poloxamer 188 with the highest average then PLGA nanoparticles then PLGA with caprolactone copolymer nanoparticles. But, the difference in zeta potential between the nanoparticles in each method of preparation is very close and non significant.

\subsection{Morphological analysis and imaging:}

Morphological analysis and imaging were performed using electron scanning microscope (SEM) which is considered the most widespread method for the analysis of shape, size, size distribution and morphology for the nanoparticles.

However, the particles are showing smooth, spherical shape with a nano size ranges between $300 \mathrm{~nm}$ to $400 \mathrm{~nm}$ for most of the particles. Furthermore, the nanoparticles are showing smooth surface with no pinholes or cracks. The nanoparticles prepared by double emulsion technique are showing more variation in size and higher poly dispersity than the particles prepared by microfluidic technique as microfluidic technique can provide a homogenous distribution of nanoparticles. Incorporation of caprolactone copolymer and poloxamer 188 to the nanoparticles have no effect on the morphological characterization of the nanoparticles [14].

\subsection{Loading and encapsulation efficiency of the insulin loaded nanoparticles.}

Encapsulation efficiency is the total amount of drug entrapped in the nanoparticles and can be calculated by dividing the amount of entrapped drug by the total amount of drug added. Encapsulation efficiency was performed by hydrolysis of the nanoparticles with $1 \mathrm{M}$ $\mathrm{NaOH}$ then measuring the released insulin with microplate reader after processing with Pierce ${ }^{\mathrm{TM}}$ BCA Protein Assay Kit. 
However, the nanoparticles prepared by double emulsion technique have slightly higher encapsulation efficiency than the nanoparticles prepared by microfluidic technique. Despite microfluidic has many advantages over the double emulsion technique but, method optimization still needed to get better encapsulation efficiency.

For the nanoparticles prepared by double emulsion technique, PLGA nanoparticles comes with the highest encapsulation efficiency with average of $52.74 \%$ which was not expected to be the highest as it was expected for PLGA with caprolactone and poloxamer to be the highest because as poloxamer can help in nanoparticles formation in the primary emulsion phase and it comes with encapsulation efficiency of $45.11 \%$ then last comes PLGA with caprolactone nanoparticles with average EE \% of $42.38 \%$.

But for the nanoparticles prepared by microfluidic technique, as expected PLGA with caprolactone and poloxamer 188 nanoparticles have the highest encapsulation efficiency with average of $44.15 \%$, then comes PLGA with caprolactone nanoparticles and PLGA nanoparticles with average encapsulation efficiency of $43.14 \% \& 40.52 \%$ respectively.

\subsection{In vitro release of insulin loaded nanoparticles.}

The main goal of this research is to control the release of insulin out of the nanoparticles in a sustained release and predictable pattern. The release of the drug out of the nanoparticles depends on the solubility of the drug, bounding to the polymers, drug diffusion and biodegradation of the nanoparticles. All these factors control the release of the drug out of the nanoparticles. In this research, we are focusing how to regulate the release by changing the polymer type by incorporation of different types of polymers to the main polymer which is PLGA and preparation technique which affects the drug diffusion and bounding forces between the polymer and the drug.

This test has been performed by incubating the formulations in phosphate buffer (PBS $\mathrm{pH} 7.4)$ at $37{ }^{\circ} \mathrm{C}$ and samples were taken at predetermined time intervals 2 hours, 1 day, 2 days, 3 days, 4 days and 7 days. The withdrawn samples were further analyzed by microplate reader using Pierce ${ }^{\mathrm{TM}}$ BCA Protein Assay Kit at $562 \mathrm{~nm}$ wavelength.

PLGA nanoparticles prepared by double emulsion technique, these particles are showing the highest burst initial release with average of $70.32 \%$ after two hours and full release of 93.25\% after seven days. By incorporating a hydrophobic polymer (caprolactone copolymer) the initial burst release decreased to $37.89 \%$ after two hours with full release of $48.48 \%$ after seven days. by additional incorporation of another hydrophobic polymer which is poloxamer 188 the initial release slightly decreased to be $36.26 \%$ after two hours with a full release of $47.07 \%$ after seven days. The initial burst release has been much lowered by the addition of hydrophobic polymers but the full release still too low which needs further improvement.

For the nanoparticles prepared by microfluidic technique, PLGA nanoparticles have initial burst release of $12.19 \%$ after two hours which was unexpected and it is much lower comparing to the PLGA nanoparticles prepared by double emulsion technique and got a full release of 57.64 after seven days. After incorporation of caprolactone copolymer the initial release decreased to $10.12 \%$ after two hours with a full release of $41.04 \%$ after seven days. by the addition of poloxamer 188 the initial release increased to $29.04 \%$ after two hours and full release of $43.45 \%$ after seven days.

Nanoparticles prepared by microfluidic technique have been showing improved pattern of release than those prepared by double emulsion technique especially after the addition of poloxamer 188 but still the full release needs some improvement as high amount of insulin still entrapped inside the nanoparticles. This might be improved by addition of hydrophilic 
polymer which can facilitate the diffusion of the drug and decrease the entrapped amount of the drug inside the nanoparticles.

\subsection{FTIR (Fourier transform infrared spectrophotometry).}

Fourier transform infrared spectrophotometry (FTIR) is used to confirm the qualitative composition of the nanoparticles. It is also used to check the compatibility of the ingredients. FTIR and confirm that there is no chemical interaction between them. FTIR works by identifying the functional groups in the samples being tested. All the ingredients of the insulin loaded nanoparticles should be encapsulated inside the PLGA matrix and this can be confirmed by the FTIR by comparing the spectrum of the nanoparticles with the ingredients which should show the same peaks as PLGA to ensure the entrapment of all ingredients inside the core of the nanoparticles.

The spectrum of PLGA nanoparticles has been plotted against insulin, PVA and PLGA. For the PLGA with caprolactone copolymer nanoparticles the spectrum has been plotted against insulin, PVA, PLGA and caprolactone copolymer. For the PLGA with caprolactone copolymer and poloxamer 188 nanoparticles the spectrum has been plotted against insulin, PVA, PLGA, Caprolactone copolymer and poloxamer 188.

All the nanoparticles are showing matching between their spectrum and PLGA spectrum which is showing a stretching peak of (C-O-C) group at $1088 \mathrm{~cm}-1$, and a methyl stretching $(\mathrm{C}-\mathrm{H})$ between 1400 and $1500 \mathrm{~cm}-1$, main peak of stretching carbonyl group (C-O-O) between 1700 and $1800 \mathrm{~cm}-1$ and stretching $(\mathrm{CH}, \mathrm{CH} 2, \mathrm{CH} 3)$ groups between 2800 and 3000 cm-1 ${ }^{[15]}$.

From the previous data we can confirm that all the ingredients of the nanoparticles are entrapped inside with no chemical interaction between them which confirm the compatibility between the ingredients.

\subsection{DSC (differential scanning calorimetry).}

Thermal analysis has been performed by differential scanning calorimetry (DSC q1000, TA instruments) to determine the thermal state of the nanoparticles and confirm the compatibility of the nanoparticles and it is very useful for the detection of any thermal interactions. That was performed by scanning them in the temperature range from 40 to 300 ${ }^{\circ} \mathrm{C}$ at a rate of $10{ }^{\circ} \mathrm{C} / \mathrm{min}$. for the PLGA nanoparticles prepared by both techniques, the thermograms were plotted against insulin, PVA, PLGA. For PLGA / caprolactone copolymer nanoparticles, the thermograms were plotted against insulin, PVA, PLGA and caprolactone copolymer and for PLGA / caprolactone copolymer / poloxamer 188 nanoparticles, thermograms were plotted against insulin, PVA, PLGA, caprolactone and poloxamer 188 for the comparison between each type of nanoparticles and its ingredients.

PLGA is showing a transition temperature (Tg) between $49{ }^{\circ} \mathrm{C}$ and $50{ }^{\circ} \mathrm{C}$. All the nanoparticles are showing the same peak as PLGA and other peaks of the ingredients disappeared in the nanoparticles thermograms which confirm the stability and compatibility of the ingredients of the nanoparticles with no thermal interactions.

\section{Conclusions and future prospects.}

Finding alternative treatment for diabetic patients instead of using multiple daily injections of insulin is what this research aims to by designing controlled release insulin nanoparticles and studying the effect of the preparation techniques and the polymers on the physiochemical properties of the nanoparticles. The insulin loaded nanoparticles prepared are in the size range between 300 and $400 \mathrm{~nm}$ with poly dispersity (PDI) not more between 0.15 and 0.45 . However, incorporation of Poly (DL-lactide-co-caprolactone) has shown decrease 
in the size average and it was decreased more after the addition of poloxamer 188 which acts as a co-emulsifier in the formulations prepared by the both techniques. Also, the microfluidic technique has shown improvement and decrease in the average size than the double emulsion technique with less variation in the size dispersion as the microfluidic technique has much better control on the size and dispersity of the nanoparticles over the double emulsion technique. All the nanoparticles are showing a negative charge on the surface with average between -6 and $-18 \mathrm{mV}$, but the negativity of the particles is lowered in the particles prepared by double emulsion technique that is because of using different organic solvents in the preparation of the nanoparticles. by examining the nanoparticles by scanning electron microscope (SEM), all the nanoparticles showed a smooth and spherical morphology with no pinholes or cracks. The chemical stability of the nanoparticles has been investigated using FTIR. All the ingredients of the nanoparticles showed compatibility and the results confirm the encapsulation of all the ingredients inside the nanoparticles. Also, thermal stability has been tested by DSC which also confirms the compatibility and full encapsulation of the ingredients inside the nanoparticles. For the loading and encapsulation efficiency all particles showed good encapsulation between $40 \%$ and $53 \%$ with no significant difference between the formulations which was unexpected as it was expected for the nanoparticles prepared by microfluidic technique to has higher encapsulation efficiency. However, the nanoparticles showed different release patterns. The PLGA nanoparticles prepared by double emulsion technique showed high initial burst release after two hours which was much lowered by the incorporation of Poly (DL-lactide-co-caprolactone) and poloxamer 188. Also, all the formulations prepared by the microfluidic technique showed improvement in the initial release by showing significant decrease in the initial burst release. However, the nanoparticles have a problem in the final release as large amount of the encapsulated insulin still entrapped inside the nanoparticles after seven days which can be improved by increasing the hydrophilicity of the nanoparticles. As a conclusion, the microfluidic technique is showing improvement in the physicochemical properties of the nanoparticles especially in the terms of size, size dispersity and release patterns.

All the characterization tests in this research of the insulin loaded nanoparticles have been performed as in vitro studies which give indication on the behavior of the nanoparticles inside the body, but further in-vivo studies should be conducted. Also, further improvement on the nanoparticles should be performed specially in the terms of loading and release patterns which can be improved by adjusting the hydrophilicity of the nanoparticles by increasing the amount of poloxamer or adding another hydrophilic polymer.

\section{References}

1. WU, Zhi Min, et al. HP55-coated capsule containing PLGA /RS nanoparticles for oral delivery of insulin . International journal of pharmaceutics , 2012, 425.1-2: 1-8.

2. HAGGAG, Yusuf, et al. Preparation and in vivo evaluation of insulin-loaded biodegradable nanoparticles prepared from diblock copolymers of PLGA and PEG . International journal of pharmaceutics , 2016, 499.1-2: 236-246.

3. Hung, L. and Lee, A. (2007). Microfluidic Devices for the Synthesis of Nanoparticles and Biomaterials. Journal of Medical and Biological Engineering, 27(1), pp.1-6.

4. Kastner, E., Verma, V., Lowry, D. and Perrie, Y. (2015). Microfluidic-controlled manufacture of liposomes for the solubilisation of a poorly water soluble drug. International Journal of Pharmaceutics, 485(1-2), pp.122-130.

5. Yan, F., Zhang, C., Zheng, Y., Mei, L., Tang, L., Song, C., Sun, H. and Huang, L. (2010). The effect of poloxamer 188 on nanoparticle morphology, size, cancer cell uptake, and cytotoxicity. Nanomedicine: Nanotechnology, Biology and Medicine, 6(1), pp.170-178. 
6. Pathak, Y. and Thassu, D. (2009). Drug delivery nanoparticles formulation and characterization. 1st ed. New York: Informa Healthcare.

7. Vladisavljević, Goran, Ruqaya Al Nuumani, and Seyed Nabavi . "Microfluidic production of multiple emulsions ." Micromachines 8.3 (2017): 75.

8. WINTER, W. T. Measurement of suspended particles by quasi -elastic light scattering , Barton E. Dahneke, Ed., Wiley, New York, 1983, 570 pp. Price: \$39.95. Journal of Polymer Science: Polymer Letters Edition, 1983, 21: 1020-1020.

9. Taylor, Robert A., et al. "Stability testing of silver nanodisc suspensions for solar applications." Applied Surface Science 455 (2018): 465-475.

10. Hwisa, N., Katakam, P., Chandu, B. and Adiki, S. (2013). Solvent Evaporation Techniques as Promising Advancement in Microencapsulation. Vedic Research International Biological Medicinal Chemistry, 1(1), p.8.

11. Zhigaltsev, I., Belliveau, N., Hafez, I., Leung, A., Huft, J., Hansen, C. and Cullis, P. (2012). Bottom-Up Design and Synthesis of Limit Size Lipid Nanoparticle Systems with Aqueous and Triglyceride Cores Using Millisecond Microfluidic Mixing. Langmuir, 28(7), pp.36333640.

12. Makadia, H. and Siegel, S. (2011). Poly Lactic-co-Glycolic Acid (PLGA) as Biodegradable Controlled Drug Delivery Carrier. Polymers, 3(4), pp.1377-1397.

13. Fernández, J., Etxeberria, A. and Sarasua, J. (2012). Synthesis, structure and properties of poly(L-lactide-co--caprolactone) statistical copolymers. Journal of the Mechanical Behavior of Biomedical Materials, 9, pp.100-112.

14. LAITINEN, Niklas; ANTIKAINEN, Osmo; YLIRUUSI, Jouko. Characterization of particle sizes in bulk pharmaceutical solids using digital image information . Aaps Pharmscitech, 2003, 4.4: 383-391.

15. Wang, Y., Li, P. and Kong, L. (2013). Chitosan-Modified PLGA Nanoparticles with Versatile Surface for Improved Drug Delivery. AAPS PharmSciTech, 14(2), pp.585-592. 